Published in Modern Nonlinear Optics. Part 3, 2nd Edn, Adv. Chem. Phys. 119, 469-524 (2001).

\title{
1 Fluctuational escape and related phenomena in nonlinear optical systems
}

\author{
I. A. KHOVANOV ${ }^{1}$, D. G. LUCHINSKY ${ }^{2,3}$, R. MANNELLA ${ }^{4,2}$, and \\ P. V. E. McCLINTOCK ${ }^{2}$ \\ ${ }^{1}$ Department of Physics, Saratov State University, Astrahanskaya 83, 410026, Sara- \\ tov, Russia \\ ${ }^{2}$ Department of Physics, Lancaster University, Lancaster LA1 4YB, UK \\ ${ }^{2}$ Russian Research Institute for Metrological Service, Ozernaya 46, 119361 Moscow, \\ Russia \\ ${ }^{4}$ Dipartimento di Fisica, Università di Pisa and Istituto Nazionale Fisica della Materia \\ UdR Pisa, Via F. Buonarroti 2, 56100 Pisa, Italy
}

\subsection{INTRODUCTION}

A characteristic feature of nonlinear science generally, and of nonlinear optics in particular, is the common necessity of having to make simplifications, and then approximations in order to solve the equations of even the simplified models. These considerations apply a fortiori to the study of fluctuation phenomena in nonlinear systems, and thus account for the increasing role being played by analogue and digital simulations, which enable the behaviour of the model systems to be investigated in considerable detail.

Several years have now passed since a contribution [1] to an earlier volume in this series illustrated some of these ideas. It was shown in particular that detailed analyses of fluctuations in model systems not only provide a deeper understanding of complex phenomena but often also pave the way to the development of new experimental techniques and new ideas of technological significance.

In this chapter, we discuss the application of simulation techniques to the study of fluctuational escape and related phenomena in nonlinear optical systems: that is, situations where a large deviation of the system from an equilibrium state occurs under the influence of relatively weak noise present in the system. We will be interested primarily in the analysis of situations where large deviations lead to new nontrivial behaviour or to a transition to a different state. The topics to be discussed have 
been selected mainly for their own intrinsic scientific interest, but also in order to provide an indication of the power and utility of the simulation approach as a means of focusing on, and reaching an understanding of, the essential physics underlying the phenomena under investigation; they also provide examples of different theoretical approaches and situations where numerical and analogue simulations have led to the development of new experimental techniques and new ideas with potential technological significance. Although the different Sections all share the same general theme - of fluctuational escape phenomena in model nonlinear optical systems - they deal with quite different aspects of the subject; each of them is therefore to a considerable extent self-contained (with Secs. 1.4 and 1.5 being exceptions, because they should be read after Sec. 1.3) and thus can be read almost independently of the others. Before considering particular systems, we review briefly the scientific context of the work and discuss in a general way the significance of escape phenomena in nonlinear optics.

The investigation of fluctuations by means of analog or digital simulation is usually found most useful for those systems where the fluctuations of the quantities of immediate physical interest can be assumed to be due to noise. The latter perception of fluctuations goes back to Einstein, Smoluchowski, and Langevin [2, 3, 4] and has often been used in optics (cf. Refs. [5, 6, 7, 8]). In nonlinear optics, the noise can be regarded as arising from two main sources. First there are internal fluctuations in the macroscopic system itself. These arise because spontaneous emission of light by individual atoms occurs at random, and because of fluctuations in the populations of atomic energy levels. The physical characteristics of such noise are usually closely related to the physical characteristics of the model that describes the "regular" dynamics of the system, i.e. in the absence of noise. In particular, the power spectrum of thermal noise and its intensity can be expressed in terms of the dissipation characteristics via the fluctuation-dissipation relations (cf. Ref. [9]) and, if the dissipation is non-retarded so that the corresponding dissipative forces (e.g. the friction force) depend only on the instantaneous values of dynamical variables, the noise power spectrum is independent of frequency, i.e. the noise is white. The model of noise as being white and Gaussian is one of the most commonly used in optics because the quantities of physical interest often vary slowly compared with the fast random processes that give rise to the noise, like emission or absorption of a photon $[5,6,7,8]$. The second very important source of noise is external: for example, fluctuations of the pump power in a laser. The physical characteristics of such noise naturally vary from one particular system to another; its correlation time is often much longer than that of the internal noise, and its effects can be large and sometimes quite unexpected (cf. Ref. [10]).

In general, the fluctuations observed in nonlinear optics are both spatial and temporal, i.e. the variations of the quantities of interest occur to a large extent independently in time and in space. However, in many cases the spatial modes in a system are well-separated: the dynamics of interest is then just that of a few dominant modes. The appearance of such modes is typical for high- $\mathrm{Q}$ active and passive optical cavities. In view of recent progress in microelectronics (quantum-dot technology, semiconductor-laser arrays, etc.), the investigation of systems with a discrete set 
of spatial structures (modes) is particularly interesting and important [11]. The amplitudes and phases of the actual modes (or other appropriate characteristics of a system that do not depend on coordinates) make a set of purely dynamical variables, and the analysis of fluctuations in a system reduces to the investigation of the kinetics of a dynamical system.

One of the most remarkable phenomena where fluctuational transitions play a key role is stochastic resonance (SR), a phenomenon in which a weak periodic signal in a nonlinear system is enhanced by an increase of the ambient noise intensity; a stronger definition requires that the signal/noise ratio (SNR) should also increase. The SR phenomenon appears to be widespread. After being introduced as a possible explanation of the earth's ice-age cycle $[12,13]$, SR has subsequently been observed or invoked in a large variety of contexts: see e.g. $[14,15,16,17,18]$ for reviews. SR has also been extensively investigated in nonlinear optical systems including lasers $[19,20,21,22]$, passive optical systems [23, 24, 25, 26], and a Brownian particle in an optical trap [27]. In this chapter, following a brief introduction to the SR phenomenon in an optical bistable system, a new form of optical heterodyning related to stochastic resonance is described, in which two high-frequency signals (input and reference signals) are applied to a bistable system. We note that the effect of noise-enhanced heterodyning was first predicted theoretically and investigated in analogue model in a broad range of parameters [28]. These investigations in turn made it possible to observe a noise-induced enhancement of heterodyning in an optical bistable device [29]. At the same time, noise-induced increase of the SNR (rather than of the signal only) can occur only in certain classes of nonlinear systems [31].

When it was first discovered, and for some time afterwards, SR seemed a rather mysterious phenomenon and a number of highly sophisticated theoretical approaches were proposed (see citations in e.g. the reviews [16, 30, 32, 33]. All these theories assumed that bistability is an essential prerequisite for the SR phenomenon to occur. Only some years later was it appreciated $[34,35]$ that a much simpler formalism linear response theory (LRT) - would suffice to describe what was often the most interesting limit in practice, where the signal was relatively small and the noise was relatively strong. An analytic theory of the more complicated effects that occur for stronger signal strengths [34, 36, 37, 38] has also been developed, and has been confirmed in considerable detail through analogue electronic experiments.

The perception of SR as a linear response phenomenon led naturally, however, to the realisation that SR can also occur without bistability [31, 39, 40] and to observation of the phenomenon in an underdamped, monostable, nonlinear oscillator [39]. In fact, it is well-known that the response of a monostable system to signals in certain frequency ranges can be strongly increased by noise, e.g. just by raising the temperature. Examples range from currents in electron tubes to optical absorption near absorption edges in semiconductors. For underdamped oscillators, a temperatureinduced shift and broadening of the absorption peaks, i.e. "tuning" by external driving due to the oscillator nonlinearity, was first discussed in [41]; complete classical and quantum theories of these effects were given in [42]. Underdamped systems were also considered in [43]. 
Describing SR in terms of a susceptibility is particularly advantageous for systems that are in thermal equilibrium, or in quasi-equilibrium. In such cases the fluctuationdissipation relations [9] can be used to express the susceptibility in terms of the spectral density of fluctuations in the absence of the periodic driving. This was used explicitly in the case of noise-protected heterodyning. It is true in general that the analysis of fluctuations is greatly facilitated by the presence of thermal equilibrium when the conditions of detailed balance and of the time reversal symmetry are satisfied [44].

However, in many cases the fluctuating systems of interest are far from thermal equilibrium. Examples include optical bistable devices [45], lasers [46, 23], pattern forming systems [47], trapped electrons which display bistability and switching in a strong periodic field [48, 49, 50], and spatially periodic systems (ratchets) which display a unidirectional current when driven away from thermal equilibrium $[51,52,53,54,55,56]$

A powerful tool for analysing fluctuations in a non-equilibrium systems is based on the Hamiltonian [57] theory of fluctuations or alternatively on a path-integral approach to the problem $[44,58,59,60,61,62]$. The analysis requires the solution of two closely interrelated problems. The first is the evaluation of the probability density for a system to occupy a state far from the stable state in the phase space. In the stationary regime, the tails of this probability are determined by the probabilities of large fluctuations.

The other problem is that of the fluctuational paths along which the system moves when a large fluctuation occurs. The distribution of fluctuational paths is a fundamental characteristic of the fluctuation dynamics, and its understanding paves the way to developing techniques for controlling fluctuations. Its importance for gaining insight into the physics of fluctuations from a dynamical perspective was recognized almost half a century ago by Onsager and Machlup [44]. A theoretical understanding, and basic techniques for treating the problem, have been developed since that time; but it was not until recently [60] that the distribution of fluctuational paths for large fluctuations was observed in an actual experiment, through an analogue simulation.

A simple qualitative idea behind the theory of large fluctuations in noise-driven systems is that such fluctuations result from large outbursts of noise that push the system far from the attractor. The probabilities of large outbursts are small, and will actually be determined by the probability of the most probable outburst of noise capable of bringing the system to a given state. This particular realisation of noise is just the optimal fluctuational force. Because a realisation (a path) of noise results in a corresponding realisation of the dynamical variable [63], there also exists an optimal path along which the system arrives to a give state with overwhelming probability. From a different perspective, optimal paths were first described for nonlinear nonequilibrium Markov systems in [57]. Using another approach, the analysis of the tails of the distribution was also done in [64], whereas the approach described above was discussed in [65] in the context of escape from a metastable state. This approach is not limited to Markov systems [42]. For systems driven by Ornstein-Uhlenbeck noise, the problem of optimal paths was discussed in $[58,66,67,68,69,70,71,72,73]$; an 
equivalent eikonal formulation was developed in $[74,75,76,77]$. The general case of Gaussian noise was discussed in [78, 79, 80]; see also Sec. 1.3. For reviews of related work on fluctuations in colored noise driven systems see [81, 82].

A brief introduction to the theory of large fluctuations is given in Sec. 1.3 together with the results of some direct observations of the optimal paths in model systems. It is very important to note that, following the first observations of optimal paths in analogue electronic models, fluctuational paths have very recently been investigated in optical systems, including measurements of the so-called prehistory probability distribution (PPD) of the radiation intensity $I$ for dropout event in a semiconductor laser [83], and the time-resolved measurement of polarization fluctuations in a semiconductor vertical-cavity surface emitting laser [84].

The preliminary analogue and numerical simulations made it possible to test fundamental tenets of the theory of large fluctuations, and thus to provide an experimental basis on which the theory could advance. At the end of Sec. 1.3 and in Sec. 1.4 we present two examples of recent advances in the theory of large fluctuations. In the first example, the time evolution of the escape flux over a barrier on a short time scale is considered. It is a problem of of fundamental importance [85] and, furthermore, of immediate practical interest given that new methods of spectroscopy with femto-second resolution have recently become available [86]. The technique of non-stationary optimal paths can be employed to solve the problem and numerical simulations verify the theoretical predictions. The striking feature predicted theoretically and demonstrated in simulations is that, for a system initially at the bottom of the well, the escape flux over the barrier on times of the order of a period of an eigen-oscillation grows in a step-wise manner, provided that friction is small or moderate. If the initial state is not at the bottom of the well, the steps at large enough times transform into oscillations. The step-wise/oscillatory evolution at short times appears to be a generic feature of a noise-induced flux.

The second example is related to the recent analytical solution $[56,87]$ of the longstanding problem of escape from a potential well in the presence of non-adiabatic periodic driving. It was shown $[56,87]$ that, over a broad range of driving field magnitudes, the logarithm of the fluctuation probability is linear in the field, and the response can therefore be characterized by a logarithmic susceptibility (LS). We evaluate the activation energies for escape, with account taken of the field-induced lifting of the time and spatial degeneracy of instantonlike nucleation trajectories. The immediate advantage of the theory is that it provides the solution of a complicated theoretical problem in a simple analytic form that describes the dependence of the "activation energy" on both the amplitude and frequency of the driving field and can be extended immediately to a periodic driving field of arbitrary form.

Analogue experiments and digital simulations confirmed that the variation of the activation energy for escape with driving force parameters is accurately described by the logarithmic susceptibility (LS). Experimental data on the dispersion are in quantitative agreement with the theory. And, again, it is interesting to note that, after the LS was investigated in analogue and numerical simulations, it was then also measured in optical experiments on a sub-micron brownian particle in a bistable three-dimensional optical trap $[88,89]$. This research emphasizes the fundamental 
importance of the logarithmic susceptibility, a new physical quantity that relates the response of the system in the absence of detailed balance to its characteristics in thermal equilibrium. It yields quantitative agreement with experiment and expresses the corrections to the "activation energy" in a simple integral form analogous to that well-known from linear response theory.

In the preceding example, analogue and numerical simulations were used to verify existing theoretical predictions. However, in reality the significance of analogue and digital simulations goes far beyond this modest role. The analogue circuit combines features of a real physical system and of the computer model and an attentive researcher can very often make important discoveries by analysing its behaviour. Perhaps the most striking example is given in the review by Kautz [90]: "In discussing analogue simulations of a rf-biased Josephson junction, performed by Levinsen and others at Berkley, Levinsen and Sullivan conceived a new type of voltage standard...”. From our own experience, examples of theory being led by the analogue simulation include the discovery of the noise-induced spectral narrowing [91] and of SR in monostable systems [31, 39], leading to extensive research by many groups and correspondingly to substantial theoretical progress.

We then report and discuss the results of recent investigations of fluctuational escape from the basins of attraction of chaotic attractors (CA). The question of noiseinduced escape from a basin of attraction of a CA has remained a major scientific challenge ever since the first attempts to generalize the classical escape problem to cover this case [92, 93, 94]. The difficulty in solving these problems stems from the complexity of the system's dynamics near a CA and is related, in particular, to the delicate problems of the uniqueness of the solution and the boundary conditions at a CA. The approach proposed here is based on the analysis of the prehistory probability distribution. It is shown in particular that both the existence and uniqueness of solution can be verified experimentally using measurements of a PPD. Moreover, using this technique and its extension [95] to measure both the optimal paths and the corresponding optimal fluctuational force, one can identify the initial conditions on a chaotic attractor and find an approximation to the energy-optimal control function of escape from a CA, thus paving the way to exciting new applications in the field of nonlinear control. One such application to the energy-optimal control of escape from the basin of attraction of CA of a periodically driven nonlinear oscillator will be described. Finally, a fluctuational escape from a Lorenz attractor, a well-known system that is of importance in modelling the dynamics of real optical systems, will be discussed.

The chapter is organized as follows: Sec. 1.2 describes an investigation of the SR phenomenon and of noise-protected heterodyning in an electronic circuit and in an optical bistable device. Sec. 1.3 discusses the results of investigation of optimal paths for large fluctuations and their relationships to the analysis of fluctuations in real optical systems. Sec. 1.4 presents two examples of recent advances in the theory of large fluctuations related to the time evolution of the escape flux over a barrier in a potential system on a short time scale, and to the non-adiabatic escape problem. The results of numerical and analogue simulations are compared with theory. Sec. 1.5 describes investigations of the escape from a CA and the applications of these 
results to the solution of the nonlinear optimal control problem. Finally, in Sec. 1.6, we summarise the results and consider future perspectives.

\subsection{STOCHASTIC RESONANCE AND NOISE-PROTECTED HETERODYNING}

\subsubsection{Introduction}

The idea of stochastic resonance (SR) was introduced by Benzi et al. [12], and Nicolis [13], who showed that a weak periodic signal in a nonlinear system can be enhanced by the addition of external noise of appropriate intensity; it was demonstrated subsequently that the same is often true of the signal-to-noise ratio (SNR) as well $[19,96]$. Recently the quest for practical applications of SR has become a subject of intensive investigation [97]. An important restriction in this respect is $[97,28]$ that the frequency of the input signal should be low compared to the characteristic frequencies of the system under study. Indeed most investigations of SR to date [20, 23, 34, 35, 98, 99, 100, 101] (see also [102] and references therein) have related to low-frequency signals driven bistable systems. The origin of the SR in such cases lies in the fact that the low-frequency driving force modulates the probabilities of fluctuational transitions $W_{n m}$ between the coexisting stable states, and hence the populations of the states, which gives rise to a comparatively strong modulation of a coordinate of the system with an amplitude proportional to the distance between the stable positions. This mechanism of strong response of a symmetric bistable system to an external forcing was first suggested by Debye [103] in the context of molecules that have several different equivalent orientations in solids and may reorient between them. Since the transition probabilities increase sharply (exponentially, for Gaussian noise) with noise intensity $D$, the efficiency of modulation and the SNR are also sharply increased. The mechanism is operative provided: (i) the stationary populations of the states are nearly equal to each other; and (ii) the frequency of the force is much smaller than the reciprocal relaxation time $t_{r}^{-1}$ of the system (see [15]). It was suggested [28], however, and demonstrated in analogue simulations, that a related phenomenon can occur when a nonlinear system is driven by two high frequency signals: if the resultant heterodyne signal is of sufficiently low frequency, both it and its SNR can be enhanced by the addition of noise.

Here, we use the ideas of SR and heterodyning to demonstrate the new phenomenon of noise-enhanced optical heterodyning in an optically bistable (OB) device driven by two modulated laser beams at different wavelengths. An optical system was chosen for the investigations for two main reasons. First, because of recent progress in optical data processing and communication [104, 105] and of possible applications of optical bistability in this context [106]: the trend to miniaturise OB devices and to reduce their threshold power [107] has highlighted the problem of controlling the signal and the SNR in optical systems. Secondly, OB systems provide an opportunity to investigate a wide range of quite general fluctuation phenomena associated 
with coexisting stable states far from thermal equilibrium. Thus the investigation of fluctuations in these systems is of fundamental interest and significance.

In Sec. 1.2.2 the fluctuations and fluctuational transitions in an OB system subject to white noise are analysed. In Sec. 1.2.3 the phenomenon of stochastic resonance in the OB system is discussed in terms of linear response theory and the corresponding experimental results are presented. In Sec. 1.2.4 we discuss theory and experimental results for the new form of optical heterodyning noise-protected with stochastic resonance. Finally, Sec. 1.2.5 contains concluding remarks.

\subsubsection{Fluctuations and fluctuational transitions in an OB system.}

1.2.2.1 Theory. A simple model that makes it possible to describe optical bistability in a variety of systems is a plane nonlinear Fabry-Perot interferometer, filled with a medium whose refractive index is intensity dependent [106]. The "slow" kinetics of a nonlinear interferometer may be often described by a Debye relaxation equation for the phase gain $\phi$, of form

$$
\begin{aligned}
\dot{\phi}+\frac{1}{\tau}\left(\phi-\phi_{0}\right) & =I_{\text {in }}(t) M(\phi)+I_{\text {ref }}(t), \\
I_{T}(t)=N(\phi) I_{\text {in }}(t), \quad N(\phi) & =N(\phi+2 \pi), \quad M(\phi)=M(\phi+2 \pi) .
\end{aligned}
$$

Here $I_{\text {in }}(t)$ is the intensity of the incident radiation, and $\phi_{0}$ is the phase of the interferometer in the dark. The functions $N(\phi)$ and $M(\phi)$ relate the intensities of the transmitted and intracavity fields to that of the incident light. The function $I_{\mathrm{ref}}(t)$ corresponds to the intensity of radiation from an additional source, which is very likely to be present in a real device to control the operating point. This description is valid in a plane wave approximation, provided that we neglect transverse effects and the intracavity build-up time in comparison with the characteristic relaxation time of nonlinear response in the system. It has been shown that the Debye approximation holds for many OB systems with different mechanisms of nonlinearity.

Let us now consider stochastic motion in an $\mathrm{OB}$ system. In general, noise in an OB system may result from fluctuations of the incident field, or from thermal and quantum fluctuations in the system itself. We shall consider the former. The fluctuations of the intensities of the input or reference signals give rise respectively to either multiplicative or additive noise driving the phase. Both types of fluctuations can be considered within the same approach [108]. Here we discuss only the effects of zero-mean white Gaussian noise in the reference signal:

$$
I_{\text {ref }}(t)=\bar{I}_{\text {ref }}+\Delta I(t), \quad\langle\Delta I(t)\rangle=0, \quad\langle\Delta I(t) \Delta I(0)\rangle=2 D \delta(t) .
$$

In this case, for a constant intensity of the input signal, $I_{\text {in }}(t)=\bar{I}_{\text {in }}=$ const, Eq. (1.1) describes the Brownian motion of the phase $\phi$ in a bistable potential

$$
U(\phi)=\frac{1}{\tau}\left(\frac{1}{2} \phi^{2}-\phi \phi_{0}\right)-\bar{I}_{\text {ref }} \phi-\bar{I}_{\text {in }} \int_{0}^{\phi} d \phi^{\prime} M\left(\phi^{\prime}\right) .
$$


Stable states can be found, for example, by graphical solution of the equation $1 / \tau\left(\phi-\phi_{0}\right)=M(\phi) \bar{I}_{i n}+\bar{I}_{\text {ref }}$ for the potential minima [42, 65], and it can be shown immediately that $\mathrm{OB}$ arises only if system is biased by a sufficiently strong external field, i.e. when it is far away from thermal equilibrium. If the noise intensity is weak, the system when placed initially in an arbitrary state will, with an overwhelming probability, approach the nearest potential minimum and will fluctuate near this minimum. Both the fluctuations and relaxation will be characterised by the relaxation time of the system $\tau_{r}$. So within a time $\sim \tau_{r}$ the system forgets about its initial state and a quasistationary distribution is formed near the stable position. It is of Gaussian shape near its maximum and of width $\propto\left(D \tau_{r}\right)^{1 / 2}$. If the noise intensity is small compared to the potential barrier height, fluctuational transitions between the stable states occur rarely and the probabilities $W_{n m}$ of transitions are given by Kramers' [109] relation

$$
W_{\mathrm{nm}} \propto \exp \left(-\Delta U_{n} / D\right)
$$

The stationary distribution over the wells is formed over a time $\sim \max \left\{W_{\mathrm{nm}}^{-1}\right\}$. For the case of white Gaussian noise this distribution has the well known form of the Gibbs distribution:

$$
p(\phi)=Z^{-1} \exp (-U(\phi) / D), \quad Z=\int d \phi \exp [-U(\phi) / D] .
$$

For small noise intensities the distribution has sharp maxima near the stable states and their populations $w_{1,2}$ are described by the balance equations

$$
\dot{w}_{1}=-W_{12} w_{1}+W_{21} w_{2}, \quad w_{2}=1-w_{1} .
$$

For arbitrary parameters of the system, $w_{1}$ and $w_{2}$ differ dramatically from each other, one of them being $\sim 1$, with the other close to zero. Within a narrow range of parameters, however, they have the same order of magnitude and one can refer to the kinetic phase transition between the two stable states: it is analogous to the first-order phase transition in an equilibrium system with a potential (in the absence of quantum fluctuations) playing the role of the generalised free energy of the system $[42,65,110]$. This is the range of parameters that is of particular interest in the present paper.

The model (1.1)-(1.5) describes stochastic motion in a general OB system for white Gaussian noise in the low noise intensity limit. We now apply this model to the description of some experimental results on fluctuations and fluctuational transitions in some particular OB devices.

1.2.2.2 Experiment In the experiments we have used two approaches. First, we have simulated the kinetics of a bistable optical system in the Debye relaxation appoximation for different forms of potential by means of electronic analogue simulation. Secondly, we have investigated the kinetics of a double-cavity membrane system (DCMS) driven by two modulated laser beams at different wavelengths. This system is known to display optical bistability [111]. 


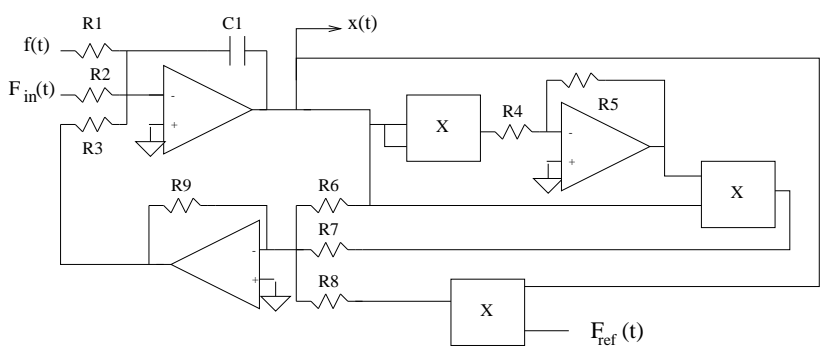

Fig. 1.1 Analog circuit used in the heterodyning simulations of [28]

An example of an electronic circuit is shown in Fig. 1.1. It is similar to the circuit used to model an OB system with a dispersive mechanism of nonlinearity [112]. The circuits were driven by noise from a feedback-shift-register noise generator and in addition, if necessary, by sinusoidal periodic forces from a pair of Hewlett-Packard Model 3325B frequency synthesizers. In the DCMS used for the optical experiments

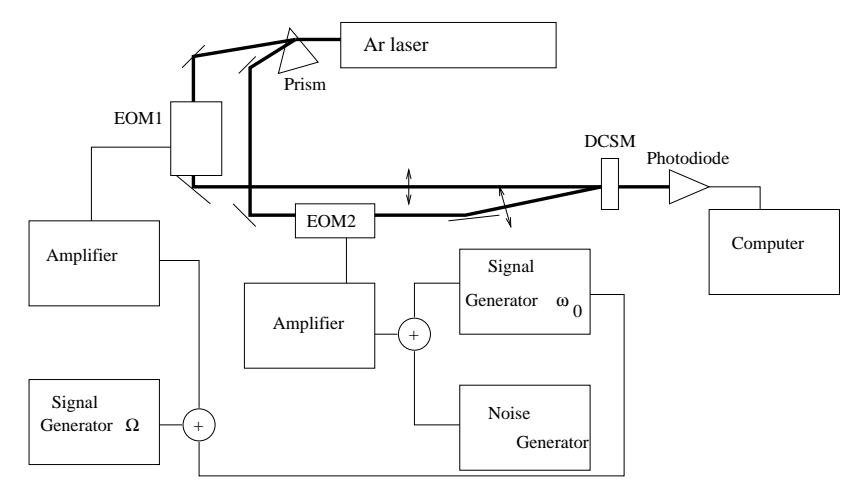

Fig. 1.2 Diagram showing the laser setup used in [29]

(see Fig. 1.2), the first resonator is formed by a membrane consisting of a thin film $(\approx 1 \mu \mathrm{m}$ thick $)$ of semiconducting GaSe single crystal, separated from a plane dielectric mirror by a metal diaphragm $\approx 500 \mu \mathrm{m}$ in diameter. The air-filled gap between the mirror and the membrane is $\approx 10 \mu \mathrm{m}$ wide and forms a second resonator. The incident beam from an argon laser, of wavelength $514.5 \mathrm{~nm}$, propagating along the normal to the mirror, provides an input signal. An additional beam of wavelength $488 \mathrm{~nm}$ from an argon laser is inclined with respect to the DCMS axis and provides a reference signal. The intensities of the laser beams are modulated by two electrooptic shutters, to which periodic signals and noise are applied. The optical bistability arises because of thermoelastic bending of the membrane caused by the $514.5 \mathrm{~nm}$ laser beam: this particular mechanism has been found to be very effective for the investigation of a variety of $\mathrm{OB}$ effects $[111,113$, ?]. The form of the periodic function $M(\phi)$ in (1.1) depends on the mechanism of thermal relaxation and the 
boundary conditions at the edge of the film; an approximate expression was found in [111] on the basis of variational analytic approaches developed for describing the thermoelasticity of shells. The phase gain $\phi$ is linear in bending and follows adiabatically the thermal relaxation of the film, thus ensuring the validity of the Debye relaxation approximation. Heating of the DCMS by the $488 \mathrm{~nm}$ reference signal is directly proportional to its intensity.

It follows from the above discussion that an indicator of applicability of the description of stochastic motion in an OB system is an activation dependence of the transition probabilities $W_{n m}$ on the noise intensity. Using level-crossing measurements (shown to be independent on the level positions), we found in our previous experiments [108] that the activation law applies over the whole range of noise intensities that we are using.

For weak noise the spectral density of fluctuations (SDF) at the output of the OB system is defined as

$$
Q(\omega)=\frac{1}{4 \pi T}\left|\int_{-T}^{+T} d t e^{i \omega t} I_{T}(t)\right|^{2} \quad T \rightarrow \infty .
$$

For small noise intensities the system spends most of the time fluctuating near the stable positions, and interwell transitions occur only occasionally. $Q(\omega)$ can then be represented as the sum of partial contributions from vibrations about the equilibrium positions $x_{n}$ weighted with the populations of the corresponding stable states $w_{n}$, and from interwell transitions. The intrawell contribution takes the form

$$
Q_{n}^{(0)}(\omega)=N^{\prime 2}\left(\phi_{n}\right) \bar{I}_{\mathrm{in}}^{2} \frac{D}{\pi} \frac{1}{U_{n}^{\prime \prime}+\omega^{2}}
$$

( $\phi_{n}$ is the value of the phase $\phi$ in the $i$ th stable state, $U^{\prime}\left(\phi_{n}\right)=0, U^{\prime \prime}\left(\phi_{n}\right)>0$ ).

One of the most important general features of fluctuations in a bistable system is the onset of a narrow zero-frequency spectral peak for parameter values lying in the range of the kinetic phase transition. This peak arises from the fluctuation-induced transitions between the stable states of the system and is of Lorentzian shape

$$
Q_{t r}^{(0)}(\omega)=\frac{w_{1} w_{2}}{\pi}\left(\bar{I}_{T 1}-\bar{I}_{T 2}\right)^{2} \frac{W_{12}+W_{21}}{\left(W_{12}+W_{21}\right)^{2}+\omega^{2}}, \quad \bar{I}_{T n} \equiv N\left(\phi_{n}\right) \bar{I}_{\mathrm{in}}
$$

The onset of this peak is closely related to stochastic resonance, which can occur if a weak periodic signal is added to the input.

\subsubsection{Stochastic resonance in an $\mathrm{OB}$ system}

For an OB system driven by a combination of the stochastic reference beam and the periodically modulated input beam $\left(I_{\mathrm{in}}(t)=\bar{I}_{\mathrm{in}}+A \cos \Omega t\right)$, the equation for the phase takes on the form

$$
\dot{\phi}+U^{\prime}(\phi)=M(\phi) A \cos (\Omega t)+\Delta I(t),
$$




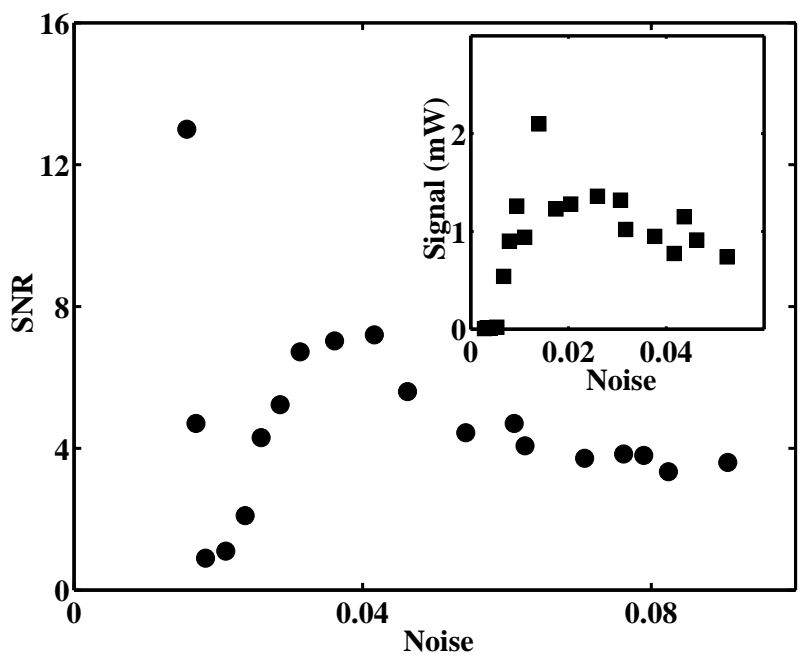

Fig. 1.3 Signal-to-noise-ratio (SNR) in the optical experiment for a signal at frequency $\Omega=3.9 \mathrm{~Hz}$ as a function of the internal noise intensity [114]. Inset: the corresponding signal amplification.

To first order in $A$ the intensity of the transmitted radiation is given by

$$
\left\langle I_{T}(t)\right\rangle=\bar{I}_{T}+A \operatorname{Re}[\chi(\Omega) \exp (-i \Omega t)]
$$

where $\chi(\Omega)$ is the susceptibility. As a result of the periodic term in the intensity of the outgoing radiation there arises a $\delta$-spike in the SDF (1.6), with the area equal to $(1 / 4) A^{2}|\chi(\Omega)|^{2}$. For low noise intensity $D$, when the system spends most of its time fluctuating about the stable states $n=1,2$, the susceptibility (like the SDF) is given by the sum of contributions from the vibrations about these states $\chi_{n}(\Omega)$ and the term $\chi_{\operatorname{tr}}(\Omega)$ that results from the periodic modulation of the populations by the force $A \exp (-i \Omega t)$.

For $\Omega \ll \tau^{-1}$ the intrawell susceptibilities correspond to quasistatic forcing, and can easily be obtained by linearising the equation of motion near the stable states. To calculate the interwell contribution to lowest order in $A / D$, one has to find corrections to the escape probabilities $W_{n m}$, which can be easily done using a path-integral formulation, solving the corresponding variational problem (see [82]), and calculating the periodic redistribution over the wells, using balance equations. The resulting expression in the case of additive noise in the OB system takes the form

$$
\chi(\Omega)=\sum_{n=1,2} M\left(\phi_{n}\right) \frac{\partial \bar{I}_{T n}}{\partial \bar{I}_{\mathrm{ref}}} w_{n}
$$




$$
+\frac{w_{1} w_{2}}{D}\left(\bar{I}_{T 1}-\bar{I}_{T 2}\right) \frac{W_{12}+W_{21}}{\left(W_{12}+W_{21}\right)-i \Omega} \int_{\phi_{1}}^{\phi_{2}} d \phi^{\prime} M\left(\phi^{\prime}\right)
$$

In general, the SDF at low frequency is a superposition of a $\delta$-function peak at the frequency $\Omega$, the zero-frequency peak, and a broad, smoothly varying background (at $\omega \tau \ll 1$ ), which is proportional to $D$ and which is small if $D$ is small.

According to $[12,13,19,96]$, the two principal features of stochastic resonance phenomena are that the signal and/or the signal-to-noise ratio $R$

$$
R=\frac{1}{4} A^{2}|\chi(\Omega)|^{2} / Q^{(0)}(\Omega) \quad(A \rightarrow 0),
$$

can be enhanced by adding noise to the system, and display resonance-like behaviour in a certain range of noise intensities. It follows from Eqs. (1.11) and (1.11) that the signal and $R$ in OB system indeed increase sharply with $D$ if the heights of the "potential barriers" satisfy $\Delta U_{1,2} \gg D$, because the probabilities of fluctuational transitions (1.3) sharply increase with noise intensity.

These particular effects have been observed experimentally [114]. A sinusoidal signal at a frequency of $3.9 \mathrm{~Hz}$ was applied to an electro-optic modulator to modulate the input signal at wavelength $514.5 \mathrm{~nm}$, while the intensity of the $488 \mathrm{~nm}$ radiation was modulated with noise. It is clearly seen from the Fig. 1.3 that the signal and $R$ (for the transmitted light intensity at wavelength $514.5 \mathrm{~nm}$ ) increase sharply in certain range of the noise amplitude $D$. Outside this range $R$ decreases with increase of $D$.

But, as mentioned above in Sec. 1.1, the mechanism of bistable stochastic resonance requires that the frequency of the input signal is much less than reciprocal relaxation time of the system.

\subsubsection{Noise-enhanced optical heterodyning.}

We now consider the case where two high-frequency fields are mixing nonlinearly in the OB system to generate a heterodyne signal. The equation of motion for the OB system takes the form

$$
\dot{\phi}+U^{\prime}(\phi)=M(\phi) A_{\text {in }}(t) \cos \left(\omega_{0} t+\psi(t)\right)+A_{\text {ref }} \cos \left(\omega_{0} t\right)+\Delta I(t),
$$

where $\omega_{0}$ is a high frequency $\left(\gg \tau^{-1}\right)$ and $A_{\text {in }}(t), \psi(t)$ are the slowly-varying amplitude and phase of the modulated input signal, respectively.

In the most interesting and practically important case, when the frequency $\omega_{0}$ is much higher than the reciprocal relaxation time of the system, simple analytical results can be obtained in the spirit of [115]. If the characteristic frequency of the modulation $\Omega \equiv \dot{\psi}$, and $\dot{A}_{\text {in }} / A_{\text {in }} \ll \tau^{-1} \ll \omega_{0}$, the response consists of a comparatively slow motion $\phi^{(\mathrm{sl})}$ with fast oscillations at frequency $\omega_{0}$ superimposed 
on it. We therefore seek a solution in the form

$$
\begin{gathered}
\phi(t)=\phi^{(\mathrm{sl})}(t)+\phi^{(1)}(t) . \\
\left.\phi^{(1)}(t)=\omega_{0}^{-1}\left[A_{\text {ref }} \sin \omega_{0} t+M\left(\phi^{(\mathrm{sl})}\right) A_{\text {in }} \sin \left(\omega_{0} t+\Omega t\right)\right)\right], \quad(\dot{\psi} \equiv \Omega)
\end{gathered}
$$

Because of the nonlinearity of $M(\phi)$, the oscillations induced by the two beams produce a slowly varying heterodyne force driving the slow motion,

$$
\begin{aligned}
\dot{\phi}^{(\mathrm{sl})}+U^{\prime}\left(\phi^{(\mathrm{sl})}\right) & =-A_{\mathrm{eff}} M^{\prime}\left(\phi^{(\mathrm{sl})}\right) \sin \Omega t+\Delta I(t) \\
A_{\mathrm{eff}} & =\frac{A_{\mathrm{ref}} A_{\mathrm{in}}(t)}{2 \omega_{0}}
\end{aligned}
$$

Thus we have reduced this problem to the form of conventional SR (see Sec. 1.2.3) with only a renormalized effective amplitude for the input signal $A_{\text {eff }}$ (cf Eq. (1.9)) and the function $M(\phi)$ replaced by its derivative $M^{\prime}(\phi)$ in the first term on the right hand side. By analogy with standard SR, the SNR for heterodyning can be characterized by the ratio $R$ of the low-frequency signal in the intensity of the transmitted radiation, given by $\frac{1}{4} A_{\text {eff }}^{2}|\chi(\Omega)|^{2}$, to the value of the power spectrum $Q^{(0)}(\Omega)$ (with $Q^{(0)}(\Omega)$ given by $(1.7),(1.8)$ ). The susceptibility of the system can be easily calculated and takes the form

$$
\begin{gathered}
\chi(\Omega)=\sum_{n=1,2} w_{n}\left(N^{\prime}\left(\phi_{n}\right)+M^{\prime}\left(\phi_{n}\right) \frac{\partial \bar{I}_{T n}}{\partial \bar{I}_{\mathrm{ref}}}\right) \\
+\frac{w_{1} w_{2}}{D}\left(\bar{I}_{T 1}-\bar{I}_{T 2}\right)\left(M\left(\phi_{1}\right)-M\left(\phi_{2}\right)\right) \frac{W_{12}+W_{21}}{\left(W_{12}+W_{21}\right)-i \Omega}
\end{gathered}
$$

Similar to what happens in conventional SR, the heterodyne signal and its signalto-noise ratio can be amplified by adding noise to the system, thus manifesting the new phenomenon of noise-enhanced optical heterodyning.

These theoretical predictions were first tested in analog simulations for Brownian motion in the symmetric Duffing potential with $M(\phi) \equiv \phi$ [28]. It was found that the heterodyne signal amplitude and corresponding $R$ could be enhanced by adding noise to the system for the cases both of white noise and of broad-band high-frequency noise (i.e. noise with a power spectrum centered near the high frequency $\omega_{0}$ with half width $\left.\Delta \omega: \tau^{-1} \ll \Delta \omega \ll \omega_{0}\right)$. The specific dependences of the renormalized amplitude of the heterodyne signal $A_{\text {eff }}$ on the amplitudes and frequency of the input and reference signals were found to be in good agreement with the theory as shown in Fig. 1.4. To investigate noise-enhanced optical heterodyning in the DCMS, the $488 \mathrm{~nm}$ reference signal was modulated periodically at frequency $\omega_{0}=2.1 \mathrm{kHz}$ and in addition by noise with a cut-off frequency of $5 \mathrm{kHz}$. The $514.5 \mathrm{~nm}$ input signal was modulated at frequencies $\omega_{0} \pm \Omega=2.1 \pm 0.0039 \mathrm{kHz}$. A heterodyne signal at frequency $\Omega=3.9 \mathrm{~Hz}$ was detected in the transmitted light intensity $I_{T}$ at wavelength 


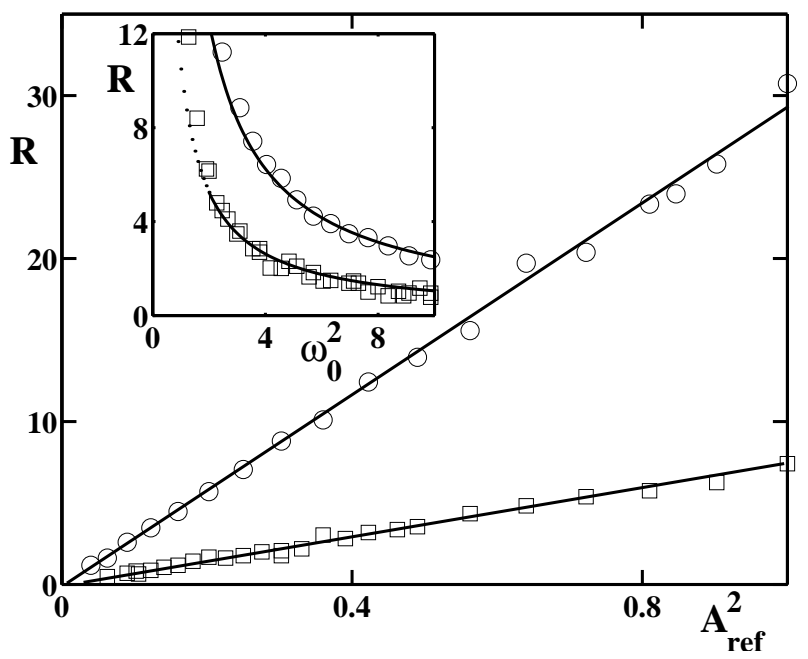

Fig. 1.4 Dependence of the signal-to-noise-ratio $R$ dependence on the squared amplitude of the reference signal, measured [28] in an analogue electronic experiment for noise intensities $D=0.015$ (circles) and $D=0.14$ (squares). The inset shows the dependence of $\mathrm{R}$ on the squared frequency $\omega_{0}$ for the same two noise intensities.

$514.5 \mathrm{~nm}$. The characteristic relaxation time $\tau_{r}$ of the DCMS measured in experiment was order of $2 \mathrm{~ms}$, thus meeting the assumption that $\Omega \ll \tau_{r}^{-1} \ll \omega_{0}$.

We have observed strong noise-induced enhancements of both the heterodyne signal (by a factor of 1000) and the signal-to-noise ratio, in Fig. 1.5. The dependence of $R$ on the noise intensity is of the characteristic reversed-N shape familiar from SR in bistable systems and consistent with the theory given above. The enhancement of the SNR occurs within a restricted range of noise intensity, as expected, and the ratio between the value of $R$ at the minimum to that at the local maximum (i.e. the maximum noise-induced "amplification" of the SNR) is $\sim 10$.

\subsubsection{Conclusions}

It will be apparent from the above discussion that the double-cavity membrane system is ideally suited to investigations of fluctuations and fluctuational transition phenomena. Stochastic resonance and huge noise-induced amplification of a heterodyne signal have been observed. We would emphasize that noise-protected heterodyning is a general phenomenon that may occur in bistable systems of various sorts, and that it may therefore be of interest for applications in engineering. 


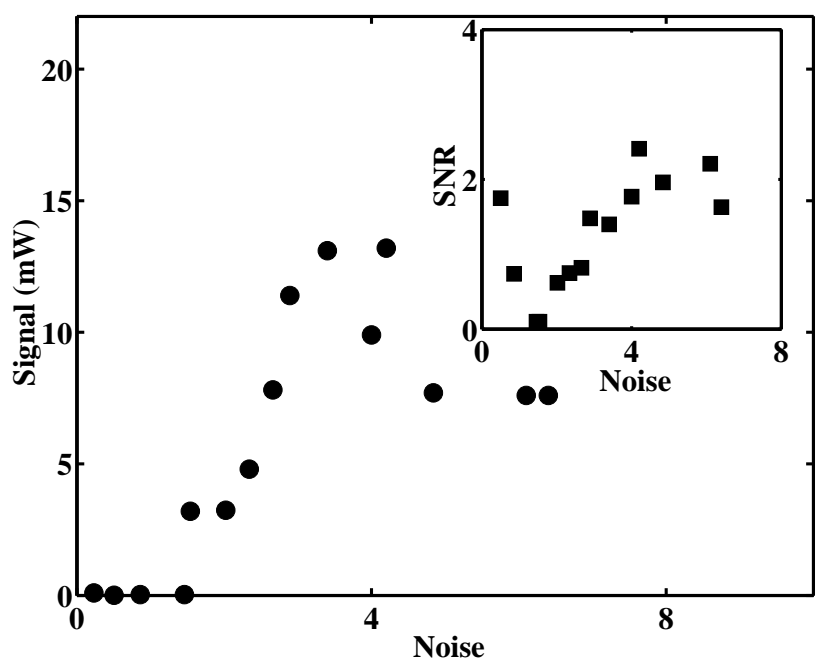

Fig. 1.5 Signal amplification in the optical heterodyning experiment, with $\omega_{0}=2.1 \mathrm{kHz}$ and $\Omega=3.9 \mathrm{~Hz}$, as a function of the internal noise intensity [29]. Inset: the corresponding signal-to-noise-ratio (SNR).

\subsection{OPTIMAL PATHS, LARGE FLUCTUATIONS, AND IRREVERSIBILITY}

\subsubsection{Introduction}

A fluctuating system typically spends most of its time in the close vicinity of a stable state. Just occasionally, however, it will undergo a much larger departure before coming back or perhaps, in some cases, making a transition to the vicinity of a different stable state. Despite their rarity, these large fluctuations are of great importance in diverse contexts including, for example, nucleation at phase transitions, chemical reactions, mutations in DNA sequences, protein transport in biological cells, and failures of electronic devices. As already mentioned above, there are many cases of practical interest where the fluctuating system is far from thermal equilibrium. Examples include lasers [46], pattern-forming systems [47], trapped electrons which display bistability and switching in a strong periodic field [48, 50], and Brownian ratchets [116] which can support a unidirectional current under nonequilibrium conditions. In general, the analysis of the behaviour of nonequilibrium systems is difficult, there being no general relations from which the stationary distribution or the probability of fluctuations can be obtained.

The most promising approach to the analysis of large fluctuations is through the concept of the optimal path $[42,57,61,65,117,118,119,120,121]$. This is the path that the system is predicted to follow with overwhelming probability during the course of the fluctuation. For many years it remained unclear how the optimal 
path - calculated as a trajectory of an auxiliary Hamiltonian system (see below) is related to the behaviour of real fluctuating systems. Recently, however, through the introduction and use of the prehistory probability distribution [60] (see also [122]), it has been demonstrated that optimal paths are physical observables that can be measured experimentally for both equilibrium [60] and nonequilibrium [123] systems. In what follows we review briefly what has been achieved and point out the opportunities that have now appeared for making rapid scientific progress in this burgeoning research field.

\subsubsection{Theory}

Consider an overdamped system driven by a periodic force $K(q ; \phi)$ and white noise $\xi(t)$, with equation of motion

$$
\begin{aligned}
& \dot{q}=K(q ; \phi)+\xi(t), \quad K(q ; \phi)=K(q ; \phi+2 \pi), \\
& \phi \equiv \phi(t)=\omega t+\phi_{0} ; \quad\left\langle\xi(t) \xi\left(t^{\prime}\right)\right\rangle=D \delta\left(t-t^{\prime}\right) .
\end{aligned}
$$

The familiar overdamped bistable oscillator driven by a periodic force provides a simple example of the kind of system we have in mind:

$$
\begin{array}{r}
\dot{q}=-U^{\prime}(q)+A \cos \omega t+\xi(t), \\
U(q)=-\frac{1}{2} q^{2}+\frac{1}{4} q^{4} .
\end{array}
$$

We consider a situation that is both nonadiabatic and nonlinear: neither $\omega$ nor $A$ need be small; only the noise intensity $D$ will be assumed small. We investigate rare fluctuations to a remote point $\left(q_{f}, \phi_{f}\right)$, coming from the metastable state within whose domain of attraction $\left(q_{f}, \phi_{f}\right)$ is located. The position of the stable state $q^{(0)}(t)$ is itself a periodic function of time,

$$
\dot{q}^{(0)}=K\left(q^{(0)} ; \phi\right), \quad q^{(0)}\left(t+2 \pi \omega^{-1}\right)=q^{(0)}(t) .
$$

The equations for optimal paths can be found using the eikonal approximation to solve the corresponding Fokker-Plank equation, or by using a path integral formulation and evaluating the path integral over the fluctuational paths in the steepest descent approximation (for details and discussion see [42, 57, 64, 65, 71, 72, 73, 117]). The optimal path of a periodically driven system corresponds to the locus traced out by the maximum in the prehistory probability density, $p_{h}\left(q, \phi \mid q_{f}, \phi_{f}\right)[60,123]$. This is the probability density that a system arriving at the point $\left(q_{f}, \phi_{f}\right)$ at the instant $t_{f}$ $\left(\phi\left(t_{f}\right)=\phi_{f}\right)$ had passed through the point $q, \phi$ at the instant $t\left(t<t_{f}\right)$. A particular advantage of this formulation is that $p_{h}$ is a physical quantity that can be measured experimentally. The approach can be extended to include the analysis of singular points in the pattern of optimal paths. 
Using the path-integral expression for the transition probability density [64], one can write $p_{h}$ in the form [60]

$$
\begin{aligned}
& p_{h}\left(q, \phi \mid q_{f}, \phi_{f}\right)=C \int_{q\left(t_{i}\right) \approx q^{(0)}\left(t_{i}\right)}^{q\left(t_{f}\right)=q_{f}} \mathcal{D} q\left(t^{\prime}\right) \delta(q(t)-q) \\
& \times \exp \left[-\frac{S[q(t)]}{D}-\frac{1}{2} \int_{t_{i}}^{t_{f}} d t^{\prime} \frac{\partial K}{\partial q}\right], \quad t_{i} \rightarrow-\infty \\
& \phi \equiv \phi(t), \quad \phi_{f} \equiv \phi\left(t_{f}\right) .
\end{aligned}
$$

Here, $C$ is a normalization constant determined by the condition

$$
\int d q p_{h}\left(q, \phi \mid q_{f}, \phi_{f}\right)=1
$$

$S[q(t)]$ has the form of an action functional for an auxiliary dynamical system with time-dependent Lagrangian $L(\dot{q}, q ; \phi)$ :

$$
S[q(t)]=\int_{t_{i}}^{t_{f}} d t L(\dot{q}, q ; \phi), \quad L(\dot{q}, q ; \phi)=\frac{1}{2}[\dot{q}-K(q ; \phi)]^{2} .
$$

In the range of small noise intensities $D$, the optimal path $q_{\text {opt }}\left(t \mid q_{f}, \phi_{f}\right)$ to the point $\left(q_{f}, \phi_{f}\right)$ is given by the condition that the action $S$ be minimal. The variational problem for $S$ to be extremal gives Hamiltonian equations of motion for the coordinate $q$ and momentum $p$ of the auxiliary system

$$
\begin{gathered}
\frac{d q}{d t}=\frac{\partial H}{\partial p}, \quad \frac{d p}{d t}=-\frac{\partial H}{\partial q}, \quad \frac{d S}{d t}=\frac{1}{2} p^{2} \\
H \equiv H(q, p ; \phi)=\frac{1}{2} p^{2}+p K(q ; \phi), \\
H(q, p ; \phi)=H(q, p ; \phi+2 \pi) .
\end{gathered}
$$

The boundary conditions for the extreme paths (1.21) follow from (1.19) and (1.20)

$$
\begin{gathered}
q\left(t_{f}\right)=q_{f} ; \\
q\left(t_{i}\right) \rightarrow q^{(0)}\left(t_{i}\right), \quad p\left(t_{i}\right) \rightarrow 0, \quad S\left(t_{i}\right) \rightarrow 0 \quad \text { for } \quad t_{i} \rightarrow-\infty .
\end{gathered}
$$

Since the Hamiltonian $H(q, p ; \phi)$ is periodic in $\phi$, the set of paths $\{q(t), p(t)\}$ is also periodic: the paths that arrive at a point $\left(q_{f}, \phi_{f}+2 \pi\right)$ are the same as the paths that arrive at the point $\left(q_{f}, \phi_{f}\right)$, but shifted in time by the period $2 \pi / \omega$. The action $S\left(q_{f}, \phi_{f}\right)$ evaluated along the extreme paths is also periodic as a function of the phase $\phi_{f}$ of the final point $\left(q_{f}, \phi_{f}\right)$. The function $S(q, \phi)$ satisfies the Hamilton-Jacobi equation

$$
\omega \frac{\partial S}{\partial \phi}=-H\left(q, \frac{\partial S}{\partial q} ; \phi\right), \quad p \equiv \frac{\partial S}{\partial q}
$$




$$
S(q, \phi)=S(q, \phi+2 \pi) .
$$

It is straightforward to see that the extreme paths obtained by solving (1.21) form a one-parameter set. It is known from the theory of dynamical systems (see for instance [124]) that trajectories emanating from a stationary state lie on a Lagrangian manifold (LM) in phase space $(q, \phi, p=\partial S / \partial q)$ (the unstable manifold of the corresponding state) and form a one-parameter set. The action $S(q, t)$ is a smooth single-valued function of position on the LM. It is a Lyapunov function: it is nondecreasing along the trajectories of the initial system in the absence of noise $\dot{q}=K(q ; \phi)$. Therefore $S(q, t)$ may be viewed as a generalised nonequilibrium thermodynamic potential for a fluctuating dynamical system [64]. The projections of trajectories in phase space onto configuration space form the extreme paths. Optimal paths are the extreme paths that give the minimal action to a given point in the configuration space. These are the optimal paths that can be visualised in an experiment via measurements of the prehistory probability distribution.

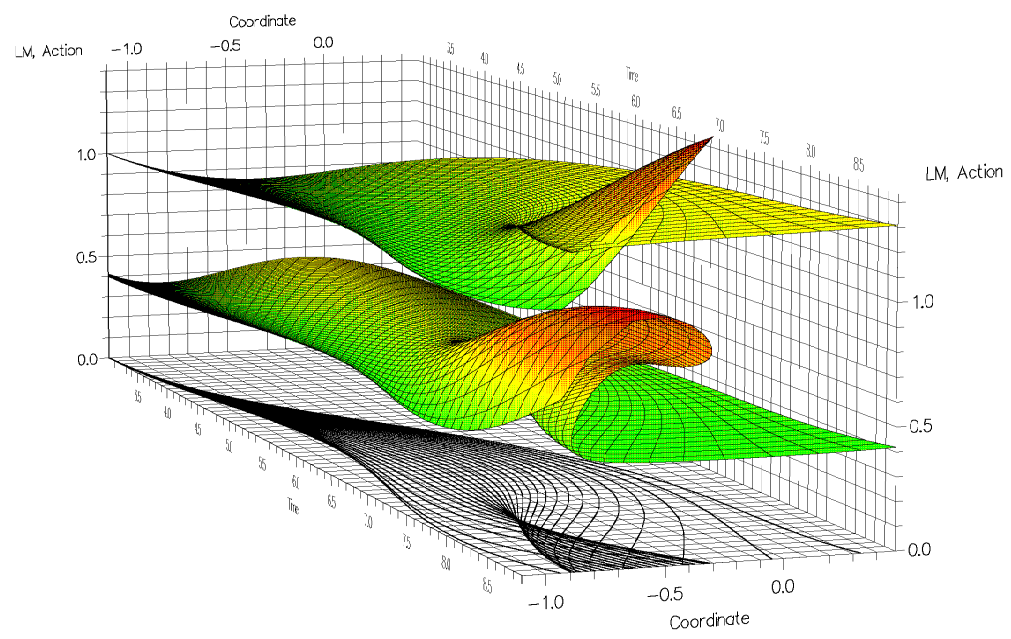

Fig. 1.6 From top to bottom: action surface; Lagrangian manifold (LM); and extreme paths calculated [80] for the system (1.17) using equations (1.21). The parameters for the system were $A=0.264$ and $\omega=1.2$. To clarify interrelations between singularities in the pattern of optimal paths, action surface, and LM surface, they are shown in a single figure, as follows: the action surface has been shifted up by one unit; and the LM has been scaled by a factor $1 / 2$ and shifted up by 0.4 .

The pattern of extreme paths, LM, and action surfaces for an overdamped periodically driven oscillator (1.17) are shown in Fig. 1.6. The figure illustrates generic topological features of the pattern in question. It can be seen from Fig. 1.6 that, although there is only one path to a point $(q, \phi, p)$ in phase space, several different 
extreme paths may come from the stationary periodic state to the corresponding point $(q, \phi)$ in configuration space. These paths cross each other. This is a consequence of the folding of the Lagrangian manifold.

A generic feature related to folding of LMs is the occurrence of caustics in the pattern of extreme paths. Caustics are projections of the folds of an LM. They start at cusp points. It is clear from Fig. 1.6 that an LM structure with two folds merging at the cusp must give rise to a local swallowtail singularity in the action surface. The spinode edges of the action surface correspond to the caustics. A switching line emanates from the cusp point at which two caustics meet. This is the projection of the line in phase space along which the two lowest sheets of the action surface intersect. The switching line separates regions which are reached along different optimal paths, and the optimal paths intersect on the switching line. The intersection occurs prior to a caustic being encountered by the optimal path. The formation of the singularities, avoidance of caustics, and formation of switching lines were analyzed numerically in [118], and a complete theory was given in [119]. Until very recently, the generic topological features of the pattern of optimal paths had not been observed in any experiment. We now describe briefly the experimental technique [123] that enables the pattern of optimal paths and its singularities to be observed, and we present and discuss some of our initial results.

\subsubsection{Experiments}

The experiments are based on analog electronic circuits designed in the usual way $[112,125]$ to model the system of interest, and then driven by appropriate external forces. Their response is measured and analysed digitally to create the statistical quantity of interest which, in the present case, was usually a prehistory probability distribution $[60,123]$. We again emphasize that such experiments provide a valid test of the theory, and that the theory should in this case be universally applicable to any system described by (1.16), including natural systems, technological ones, or the electronic models studied here. Some experiments on a model of (1.17) are now described and discussed as an illustrative example of what can already be achieved.

The model was driven continuously by external quasi-white noise from a noise generator and by a periodic force from a frequency synthesiser. The fluctuating voltage representing $q(t)$ was digitized and analysed in discrete blocks of 32768 samples using a Nicolet NIC-1180 data-processor. The input sweeps were triggered by the frequency synthesiser so that information about the phase of the periodic force could be retained. Whenever $q(t)$ entered a designated square centred on a particular $\left(q_{f}, \phi_{f}\right)$ value, the immediately preceding part of the trajectory was collected and stored; in cases where relaxation trajectories were also of interest, the immediately following part of the trajectory was preserved too. The trajectories that had arrived in any chosen square could subsequently be ensemble-averaged together to create the prehistory probability distribution $p_{h}\left(q, \phi \mid q_{f}, \phi_{f}\right)$ corresponding to the chosen $\left(q_{f}, \phi_{f}\right)$, with or without the relaxational tail back towards the stable state.

Because the fluctuations of interest were - by definition - rare, it was usually necessary to continue the data acquisition process for several weeks in order to build 


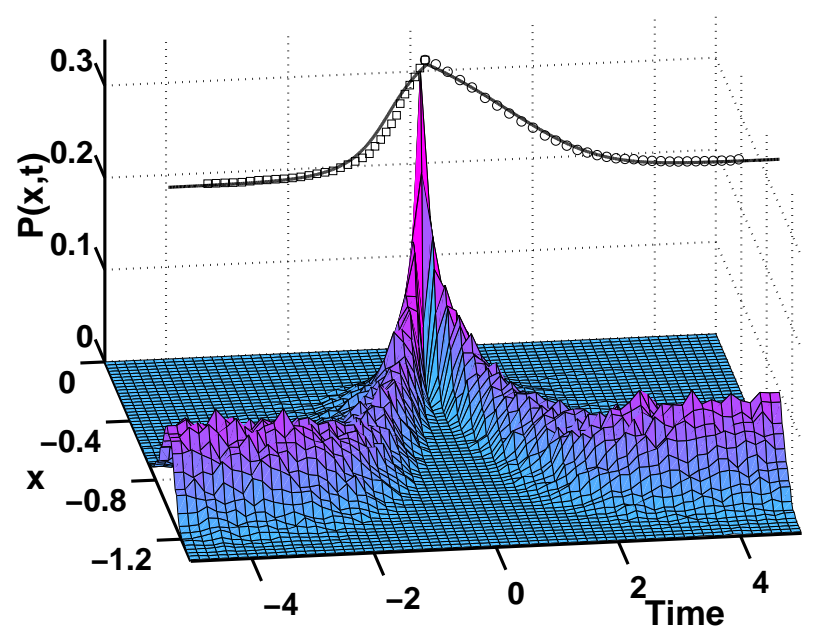

Fig. 1.7 The prehistory probability density $p_{h}\left(x, t ; x_{f}, 0\right)$ [60] for (1.17), measured [62] for $A=0$ in the analog electronic experiment for a final position $x_{f}=-0.30$ with $D=$ 0.0701 .

up acceptably smooth distributions. For this reason, the analysis algorithm was designed to enable trajectories to several termination squares (not just one) to be sought in parallel: an $8 \times 8$ matrix of 64 adjacent termination squares, each centred on a different $\left(q_{f}, \phi_{f}\right)$ was scanned.

Experimentally measured $p_{h}$ for the system (1.17) for two qualitatively different situations are shown in Figs. 1.7 and 1.8. It is immediately evident: (i) that the prehistory distributions are sharp and have well defined ridges; (ii) that the ridges follow very closely the theoretical trajectories obtained by solving numerically the equations of motion for the optimal paths, shown by the full curves on the top-planes. It is important to compare the fluctuational path bringing the system to $\left(q_{f}, \phi_{f}\right)$ with the relaxational path back towards the stable state in thermal equilibrium, Fig. 1.7, and away from it, Fig. 1.8. Fig. 1.7 plots the distribution for the system (1.17) in thermal equilibrium, i.e. $A=0$. The ridges of a distribution are compared with the calculated fluctuational and relaxational paths at the top of the figure. The time reversal symmetry [44] between these paths can be clearly seen. Fig. 1.8 plots the $p_{h}$ and the ridges of a distribution recorded for the special situation that arises when the termination point lies on the switching line [123]. In figure, the time dependent stable and unstable states bear $x=-1$ and $x=0$ are shown by dashed lines on the top. The data are compared to the (theoretical) fluctuational paths, calculated from (1.21), shown as full lines. It can be seen: that there are two distinct paths via which the system can arrive at $\left(q_{f}, \phi_{f}\right)$ but only one relaxational path taking it back to the stable state. Unlike the behaviour expected and seen [62] in equilibrium systems, neither of the fluctuational paths is a time-reversed image of the relaxational one. 


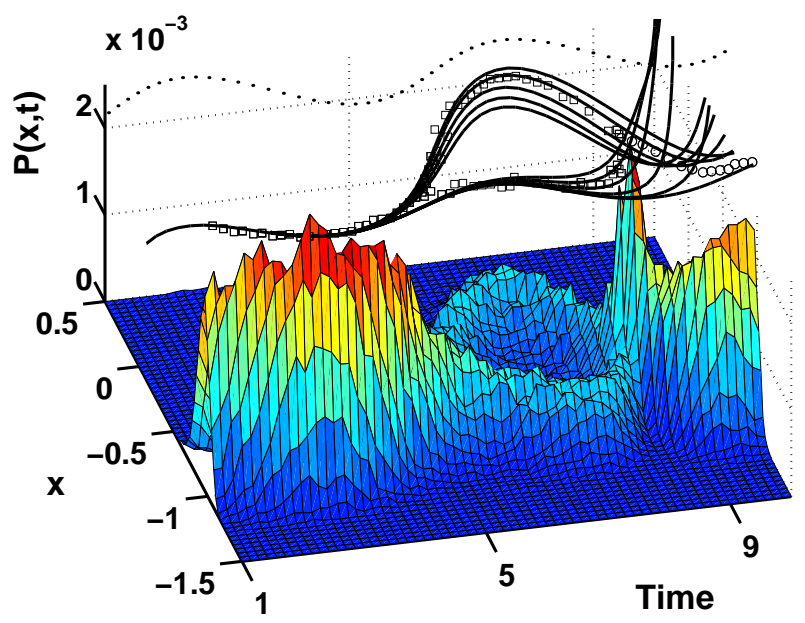

Fig. 1.8 Fluctuational behaviour measured and calculated for an electronic model of the nonequilibrium system (1.17) with $A=0.264, D=0.012$. The main figure plots the prehistory probability density $p_{h}\left(x, t ; x_{f}, 0\right)$ and posthistory distribution to/from the remote state $x_{f}=-0.63, t=0.83$, which lies on the switching line. In the top-plane, the fluctuational (squares) and relaxational (circles) optimal paths to/from this remote state were determined by tracing the ridges of the distribution [62].

Note that there are two equally probable fluctuational paths to arrive on a switching line, they form a so called corral [123].

Although the system (1.17) is relatively simple, it describes very well the fluctuational dynamics of many real physical systems. In particular, a behaviour qualitatively similar to the one shown in Fig. 1.7 was observed recently in the experiments with semiconductor lasers [83, 84].

In the work by Hales and co-authors [83] the prehistory distribution was observed experimentally using a semiconductor laser with optical feedback. Near the solitary threshold, the system was unstable: after a period of nearly steady operation, the radiation intensity decreased; then it recovered comparatively quickly, growing to regain its original value; decreased again; and the cycle repeated. In the experiment, the output intensity was digitized with $1 \mathrm{~ns}$ resolution. The $p_{h}$ obtained in [83] from 1512 events is shown in Fig. 1.9. The results were compared with the results of numerical simulation for the system (1.17).

In the work by Willemsen and co-authors [84] the three Stokes polarization parameters were studied during polarization switches in a vertical-cavity semiconductor laser. It was demonstrated that when the linear part of the absorptive anisotropy is close to zero [126], the laser is bistable and switches stochastically between two polarisations [127]. The analysis of large fluctuations of polarizations in this system [84] reveals what authors have called a "stochastic inversion symmetry" (see 


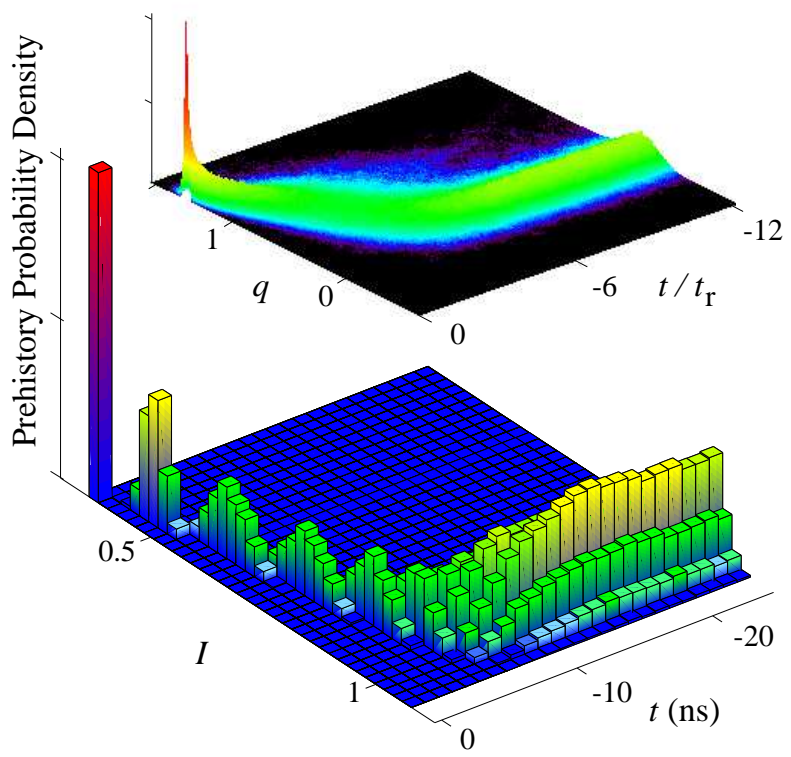

Fig. 1.9 Bottom: The prehistory probability distribution of the radiation intensity $I$ (in arbitrary units) for dropout events in a semiconductor laser. Top: The PPD for a Brownian particle, obtained from simulations [83].

Fig. 1.10), which is analogous to the time reversal symmetry observed for the model (1.17) and shown in Fig. 1.7.

\subsubsection{Optimal paths on a finite time range, and conclusions}

The previous discussion, and the results of $[62,123,128,129,130,131]$ among others, show that our analog electronic technique makes it possible to test fundamental tenets of fluctuation theory, and thus provide an experimental basis on which the theory can advance. We can investigate the pattern of optimal paths for thermally nonequilibrium systems and reveal its singularities including, in particular, switching lines and strong (nonanalytic in the noise intensity) smearing of the prehistory probability distribution near cusp points. The particular system we have investigated has the least number of degrees of freedom necessary to observe these singularities, and therefore it is most appropriate for analysis in these initial investigations. The approach that we have described is in principle applicable to any nonequilibrium system, and we believe it will be found useful in a wide range of applications. 


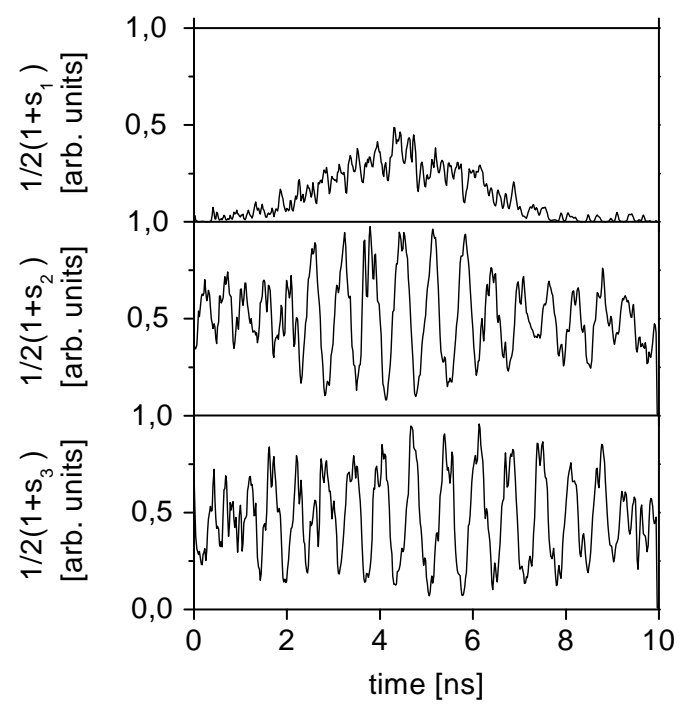

Fig. 1.10 Time-resolved measurements of a very large polarisation fluctuation, where the size of the fluctuation is about half (in fact, 45\%) of that of a complete polarisation switch [84]. $s_{1}, s_{2}$ and $s_{3}$ are the normalized Stockes parameters representing the polarization state on the Poincaré sphere [84].

It should also be clear that the structures predicted by the theory are indeed observed in real systems. Reasoning along these lines, researchers have recently started predicting peculiar features which should be observable in real systems, on the basis of the topology of corresponding Lagrangian manifold. These include features predicted on the assumption that the optimal path (and the corresponding fluctuation in the real system) takes place over a finite time range [132, 133, 134, 135, 136].

One of the most striking effects predicted on this basis is perhaps what occurs in noise induced escape from a metastable well on a time scale preceding the formation of a quasi-equilibrium distribution within the metastable part of the potential (see $[135,136]$ for more details), which we now review briefly.

In his seminal work [109], Kramers considered the noise-induced flux from a single metastable potential well i.e. he considered a Brownian particle

$$
\begin{aligned}
& \ddot{q}+\Gamma \dot{q}+d U / d q=f(t), \\
& \langle f(t)\rangle=0, \quad\left\langle f(t) f\left(t^{\prime}\right)\right\rangle=2 \Gamma T \delta\left(t-t^{\prime}\right),
\end{aligned}
$$

which was put initially at the bottom of a metastable potential well $U(q)$ and then he calculated the quasi-stationary probability flux beyond an absorbing barrier. There have been many developments and generalizations of the Kramers problem (see 
$[137,138]$ for reviews) but both he and most of those who followed him considered just the quasi-stationary flux, i.e. the flux established after the formation of a quasistationary distribution within the well (up to the barrier). The quasi-stationary flux is characterized by a slow exponential decay, an Arrhenius dependence on temperature $T$, and a relatively weak dependence on friction $\Gamma$ :

$$
J_{q s}(t)=\alpha_{\text {escape }} \mathrm{e}^{-\alpha_{\text {escape }} t}, \quad \alpha_{\text {escape }}=P \mathrm{e}^{-\frac{\Delta U}{T}},
$$

where $P$ depends on $\Gamma$ and $T$ in a non-activation manner.

But how does the flux evolve from its zero value at the initial moment to its quasi-stationary value at time-scales exceeding the time $t_{f}$ for the formation of quasi-equilibrium? It is obvious that the answer may depend on initial conditions. The most natural are those corresponding to the stable stationary state of the noisefree system i.e. $\left(q=q_{\text {bottom }}, \dot{q}=0\right)$ where $q_{\text {bottom }}$ is the coordinate of the bottom of the potential well. We assume such an initial state here. If the noise is switched on suddenly (e.g. if the thermal isolation of a frozen system is broken) then the time evolution of the escape flux from the noise-free metastable initial state is highly relevant. It might seem natural that the evolution from zero to the quasi-stationary value should be smooth. Such an assumption might also seem to have been confirmed recently by Schneidman [139] who found that, for both the strongly underdamped and overdamped cases, the escape flux from a single metastable well grows with time $t$ smoothly, at $t \sim t_{f}$. But does this exhaust the problem? We can prove theoretically, and demonstrate experimentally, that there are some generic situations when the escape flux behaves in a quite different manner.

Our prediction are based, as mentioned, on an extensive use of the method of optimal fluctuation within which an escape rate is sought in the form

$$
\alpha_{\text {escape }}=P \mathrm{e}^{-\frac{S}{T}}
$$

where the action $S$ does not depend on $T$; the prefactor $P$ does depend on $T$, but relatively weakly. The action $S$ is related to a certain optimal fluctuation which, in turn, corresponds to the most probable escape path (MPEP).

The quasi-stationary flux is formed by optimal fluctuations which bring the system from the bottom of the well to the saddle during an optimal time

$$
t_{\mathrm{opt}} \sim \frac{1}{\min \left(\Gamma, \omega_{0}\right)} \ln \left(\frac{\Delta U}{T}\right)
$$

where $\omega_{0}$ is the frequency of eigenoscillation in the bottom of the well.

At much shorter time-scales, $t \ll t_{\mathrm{opt}}$, the flux is necessarily formed by optimal fluctuations strongly differing from those of duration $t_{\mathrm{opt}}$, and the smaller $t$ the more marked this difference becomes. Thus, in the range (1.27), $S$ depends on $t$. Moreover, it can be shown rigorously that if $\Gamma<\Gamma_{c}$ where $\Gamma_{c}$ is typically equal to $2 \omega_{0}$, then $S(t)$ is a step-wise function: see the example in Fig. 1.11. The vertical and horizontal positions of the center of the step $S(t)$ number $n$ (counted from the left) equal respectively $\Delta U \omega_{0} /(n \pi \Gamma)$ and $n \pi / \omega_{0}$, provided $n \pi \Gamma \ll \omega_{0}$. Generally, 


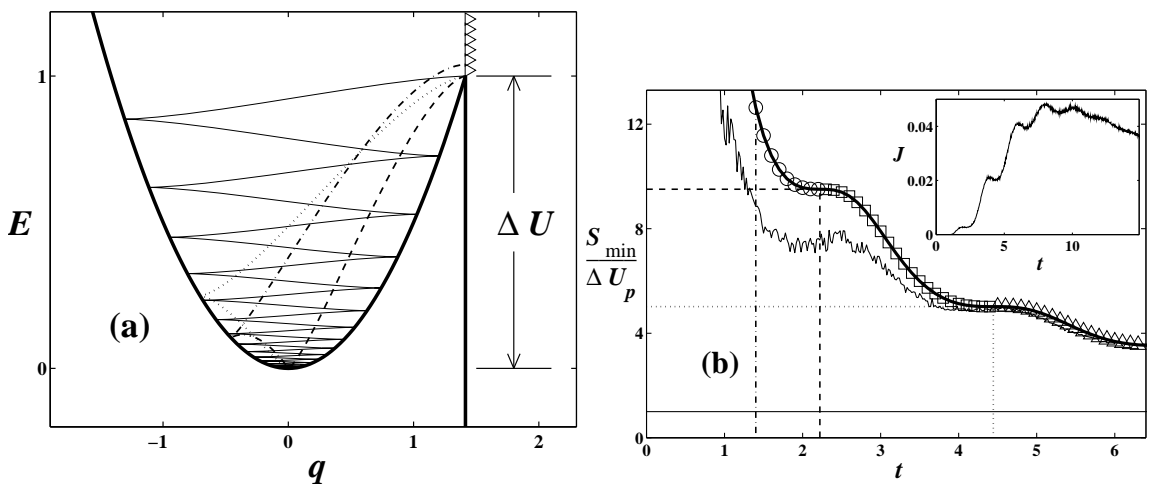

Fig. 1.11 (a) Examples of MPEPs (plotted in the energy-coordinate plane $E-q$ where $\left.E=\dot{q}^{2} / 2+U(q)\right)$ to escape from the bottom of the metastable well $U(q)=q^{2} / 2$ with $q<\sqrt{2}$ (thick solid line) to beyond the barrier at $q=\sqrt{2}(U(q)=-\infty$ at $q>\sqrt{2}$, which is equivalent to the absorbing wall indicated by triangles), for $\Gamma=0.05$; (b) the corresponding theoretical (thick solid line) and experimental (thin jagged line) dependences of the action $S$ on the escape time $t$. Circles, squares and triangles indicate bits corresponding to respectively 0,1 and 2 turning points in the MPEP. The dashed and dotted lines indicate: in (b) the 1st and 2nd inflection point with $d S / d t=0$; and in (a) the corresponding MPEPs. The thin solid line shows: in (b) the large-time asymptotic level $S=\Delta U$; and in (a) the corresponding MPEP (which is the time-reversal of the noise-free trajectory from the top of the barrier into the bottom of the well). The dash-dotted line shows in (a) the MPEP corresponding to some arbitrarily chosen time $t=4.51$ (see (b)) and demonstrates, in particular, that the escape velocity is generally non-zero. The inset shows the experimental dependence of the flux on time, for $T=\Delta U[135,136]$.

when the shape of the potential well departs from parabolicity, the equalities turn into approximations. Thus, in the range (1.27), $J$ depends exponentially strongly both on $\Gamma$ and on $t$ (c.f. the inset of Fig. 1.11).

\subsection{LOGARITHMIC SUSCEPTIBILITY}

A very good example of the usefulness of the concept of the optimal path is the idea of the logarithm susceptibility (LS) [56, 87, 140].

Underlying the theory of the LS $[56,87]$ is the realization that, although the motion of the fluctuating system is random, large rare fluctuations from a metastable state to a remote state, or during escape, take place in an almost deterministic manner: the system is overwhelmingly most likely to move along a particular trajectory known as the optimal path (see $[42,57,64,69,120]$ and references therein). The effect of a comparatively weak field on the escape probability can therefore be understood in terms of the work that the field does on the system as it moves along the optimal path. One may expect this work to be related to the field-induced change in the 
activation energy $R$ for the corresponding large fluctuation. This change is linear in the field, provided that the field-induced change of the optimal path itself is negligible. It follows from these arguments that in the case of periodic driving $F(t)=\sum_{k} F_{k} \exp (i k \Omega t)$, the leading-order correction $\delta R$ to the activation energy of escape is

$$
\begin{aligned}
& \delta R=\min _{t_{c}} \delta R\left(t_{c}\right), \delta R\left(t_{c}\right)=\sum_{k} F_{k} \tilde{\chi}(k \Omega) e^{i k \Omega t_{c}}, \\
& \tilde{\chi}(\Omega)=-\int_{-\infty}^{\infty} d t \dot{q}^{(0)}(t) e^{i \Omega t}, \dot{q}^{(0)}=U^{\prime}\left(q^{(0)}\right) .
\end{aligned}
$$

Here, $\tilde{\chi}(\Omega)$ is the LS for escape. It is given $[56,87]$ by the Fourier transform of the velocity along the most probable escape path $q^{(0)}(t)$ in the absence of driving $(F(t)=0)$. The path $q^{(0)}(t)$ is an instanton [122]: it starts for $t \rightarrow-\infty$ at the metastable minimum $q_{s}$ of the potential $U(q)$ and goes for $t \rightarrow \infty$ to the top $q_{u}$ of the potential barrier over which the particle escapes. The minimization over $t_{c}$ corresponds to choosing the position of the center of the instanton so as to maximize the work the field $F(t)$ does on the system along the escape path $q^{(0)}\left(t-t_{c}\right)$. We have already noted that, for Markov systems in thermal equilibrium, optimal fluctuational paths are the time-reversed relaxational paths in the absence of noise [44, 130, 141]. Unlike the standard linear susceptibility [9] which, on causality arguments, is given by a Fourier integral over time from 0 to $\infty$, the $\operatorname{LS} \tilde{\chi}(\Omega)$ is given by an integral from $-\infty$ to $\infty$. The analytic properties of $\tilde{\chi}(\Omega)$ therefore differ from those of the standard susceptibility, and in particular their high-frequency asymptotics are qualitatively different. The standard susceptibility for damped dynamical systems decays as a power law for large $\Omega$ (e.g., as $1 /\left[U^{\prime \prime}\left(q_{s}\right)-i \Omega\right]$, for the model of damped Duffing oscillator). In contrast, from (1.28) the LS decreases exponentially fast,

$$
\tilde{\chi}(\Omega)=M e^{-|\Omega| \tau_{p}}, \quad \tau_{p}=\min \left|\operatorname{Im} \int d q / U^{\prime}(q)\right| .
$$

Here, the integral is taken from any point in the interval $\left(q_{s}, q_{u}\right)$ to the (complex) position $q_{p}$ of the appropriate singularity of $U^{\prime}(q)$. Note that $\dot{q}^{(0)}\left(t-t_{c}\right)$ for given real $t_{c}$ has a pole or a branching point at $\operatorname{Im} t=\tau_{p}$. The prefactor $M$ depends on the form of $U(q)$ near $q_{p}$ and can be obtained in a standard way. In particular, for a polynomial potential $\left(\left|q_{p}\right| \rightarrow \infty\right)$ with $U(q)=C q^{n} / n$ for $|q| \rightarrow \infty$, we have

$$
|M|=2 \pi|\Omega / C|^{\nu}|\nu|^{\nu+1} / \nu !, \nu=1 /(n-2) .
$$

This expression applies also for finite $\left|q_{p}\right|$, with $U(q) \approx C / \mu\left(q-q_{p}\right)^{\mu}$ for $q \rightarrow q_{p}$, if $n$ in (1.30) is replaced by $-\mu$ : note that $|M|$ then decreases with increasing $\Omega$.

To test these predictions, we used an analog electronic model [112] of the overdamped motion of a Brownian particle in the double-well Duffing potential. We drove it with zero-mean quasi-white Gaussian noise from a shift-register noise generator, digitize the response $q(t)$, and analysed it with a digital data processor. We also 


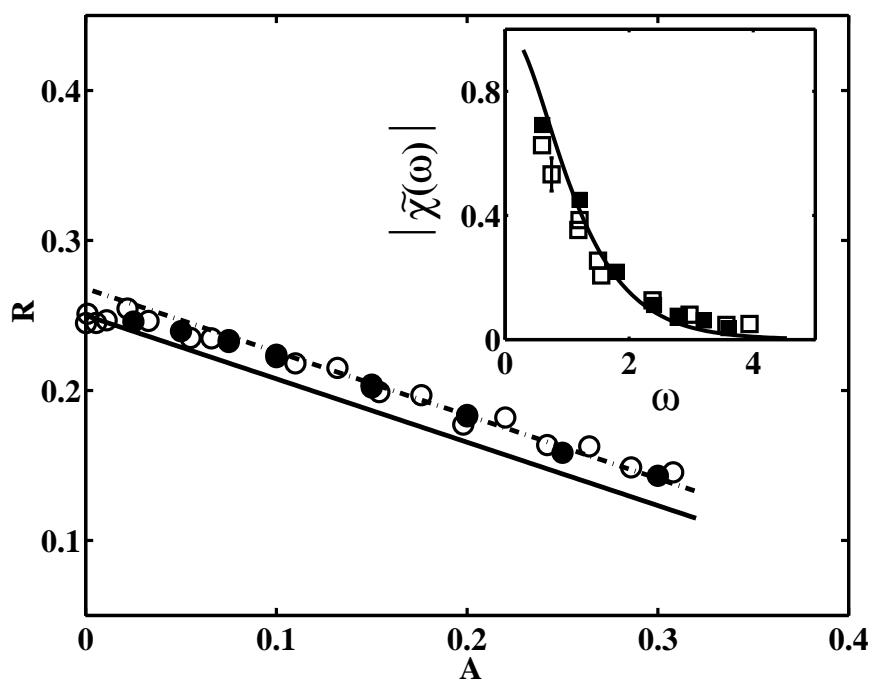

Fig. 1.12 The dependence of the activation energy $R$ on the amplitude $A$ of the harmonic driving force $F(t)=A \cos (1.2 t)$ as determined [140] by electronic experiment (filled circles), numerical simulations (open circles) and analytic calculation (solid line) based on (1.28) for an overdamped Duffing oscillator $U(q)=-q^{2} / 2+q^{4} / 4$; the dash-dot line, drawn parallel to the full curve, is a guide to the eye. The inset shows the absolute value of the LS of the system $|\tilde{\chi}(\Omega)|(1.28)$ measured (filled and open squares for experiment and numerical simulation, respectively) and calculated (full curve) as a function of frequency $\Omega$ using (1.29) with $\tilde{\chi}(0)=-1$ and $\tau_{p}=\pi / 2, M=-(1+i)(\pi \omega)^{1 / 2}$ in (1.29).

carried out a complementary digital simulation [142]. Numerical simulations in the case of small damping are currently in progress: preliminary results indicate a resonant behaviour of the LS. The analog and digital measurements of $R$ involved noise intensities in the ranges $D=0.028-0.036$ and $D=0.020-0.028$ respectively; the lowest (real time [112]) driving frequency used was $460 \mathrm{~Hz}$. The results are plotted in Fig. 1.12. The major observation is that, as expected, $R$ is indeed linear in the force amplitude ( $R=1 / 4$ for $A=0$ ). The slope yields the absolute value of the LS. Its frequency dependence, a fundamental characteristic of the original equilibrium system, is compared with the theoretical predictions (1.29) in the inset of Fig. 1.12.

The LS theory was applied recently to the localization of a Brownian particle in a three-dimensional optical trap [89]: a transparent dielectric spherical silica particle of diameter $0.6 \mu \mathrm{m}$ suspended in a liquid [88]. The particle moves at random within the potential well created with a gradient three-dimensional optical trap - a technique widely used in biophysical studies. The potential was modulated by a biharmonic force. By changing the phase shift between the two harmonics it was possible to 
localize particle in one of the wells in very good quantitative agreement with the predictions based on the LS.

\subsubsection{Conclusions}

It is evident from the above discussion that the theory of the optimal paths provides a deep physical insight into the dynamics of fluctuations and is in good agreement both with the results of analog and numerical simulations and with the results of the experiments in optical systems. It has now become possible to use the prehistory formulation [60] as a basis for experiments on fluctuational dynamics. The work on Markov systems presented in this section has already verified several longstanding theoretical predictions, including symmetry between the growth and decay of classical fluctuations [44], the breaking of this symmetry under non-equilibrium conditions [57, 59, 65, 118], the relationship between lack of detailed balance and onset of singularities in the pattern of optimal paths, as well as the character of these singularities $[119,120,121,123,143]$, including occurrence of switching between optimal paths and critical broadening of the paths distribution. It has now become possible to apply this theory and the corresponding experimental methods to the analysis of the fluctuational dynamics in optical systems and to develop new methods of controlling them.

\subsection{CHAOTIC ESCAPE AND THE PROBLEM OF OPTIMAL CONTROL}

One of the main problem in the dynamics of optical systems is that of controlling the system dynamics [144]. The difficulties in solving such a problem depend on many factors. A typical optical system is characterised by the phenomenon of multistability $[145,146,147,148]$, i.e. the co-existence of a relatively small number of distinct dynamical regimes that are defined by the initial conditions. Because real optical systems are always subject to random fluctuations $[46,146]$, spontaneous transitions of the system take place from one regime to another. It is obviously desirable to be able to control these transitions. Moreover, in optical systems non-regular oscillations are often observed which are chaotic and can be described by the theory of deterministic chaos [144, 146, 148]; such non-regular oscillations in the phase space of the system can be characterised by a chaotic attractor. The transformation of the system dynamics from a chaotic regime to a regular regime is also an interesting problem in dynamical control. In solving it for real systems, it is essential to take into account of fluctuations.

The need to be able to control chaos has attracted considerable attention recently. Methods already available include a variety of minimal forms of interaction [149, $150,151,152,153,154]$ and methods of strong control $[155,156]$ which necessarily require a large modification of the system's dynamics, for at least a limited period of time. For example, in $[157,158]$ the procedure of controlling chaos by means of minimal forms of interaction (of saddle cycles stabilization) is realized for different laser systems. 
At the same time the energy-optimal directing of the motion away from a chaotic attractor (CA) to another coexisting attractor has remained an important unsolved problem of long standing. Its solution would be an important extension of the range of model-exploration objectives (c.f. [155] and [153]) achievable through minimal control techniques and has a variety of applications for controlling the dynamics of multistable optical systems [146].

In this section the application of the optimal path approach to the problem of escape from a non-hyperbolic and from a quasi-hyperbolic attractor is examined. We discuss these two different types of chaotic attractor because it is known [159] that noise does not change very much the structure and properties of quasi-hyperbolic attractors, but that the structure of non-hyperbolic attractors is abruptly changed in the presence of noise, with a strong dependence on noise intensity. Note that for optical systems both types of chaotic attractor [160,161,162] (non-hyperbolic and quasi-hyperbolic) are observed, but a non-hyperbolic attractor is much more typical.

\subsubsection{Escape from a non-hyperbolic attractor}

1.5.1.1 Introduction to the optimal control problem Consider a system of the form

$$
\dot{x}=f(x, u, t),
$$

with the state variable $x \in R^{n}$, and an admissible control function $u \in R^{m}$ in the control set $U$. Assume that it is desired to transfer the system from the state $X_{0}=x\left(t_{0}\right)$ to the terminal state $X_{1}=x\left(t_{1}\right)$ in such a way that the ("cost") functional

$$
J=\min \int_{t_{0}}^{t_{1}} f_{0}(x, u, t) d t
$$

is minimized, with $t_{1}$ unspecified. Let $(u(t), x(t))$ be a solution of this problem. Then there exist continuous piece-wise differentiable functions $y_{0}(t), \ldots, y_{n}(t)$ which are not simultaneously zero and which satisfy together with the functions $x_{i}(t)$ the differential equations (see e.g. [163])

$$
\begin{gathered}
\dot{x}_{i}=\frac{\partial H}{\partial y_{i}} \\
\dot{y}_{i}=-\frac{\partial H}{\partial x_{i}}
\end{gathered}
$$

with the Hamiltonian

$$
H\left(x_{1}, \ldots, x_{n} ; y_{0}, \ldots, y_{n} ; u(t), t\right)=\sum_{i=0}^{n} y_{i} f_{i}\left(x_{1}, \ldots, x_{n} ; u(t), t\right)
$$

An optimal control function $u(t)$ maximizes $H$ at each instant. $H$ is a continuous function of the time and one has $H\left(t_{1}\right)=0$. If the functions $f_{i}, i=0, \ldots, n$ do not depend on time explicitly, then $H$ is a constant and equal to zero.

It can be seen that the solution of the problem of the energy-optimal guiding of the system from a chaotic attractor to another coexisting attractor requires the 
solution of the boundary value problem (1.33), (1.34) for the Hamiltonian dynamics. The difficulty in solving these problems stems from the complexity of the system dynamics near a CA and is related, in particular, to the delicate problems of the uniqueness of the solution, its behaviour near a $\mathrm{CA}$, and the boundary conditions at a CA.

Below we show how the energy-optimal control of chaos can be solved via a statistical analysis of fluctuational trajectories of a chaotic system in the presence of small random perturbations. This approach is based on an analogy between the variational formulations of both problems (see e.g. [164]): the problem of the energyoptimal control of chaos and the problem of stability of a weakly randomly perturbed chaotic attractor. One of the key points of the approach is the identification of the optimal control function as an optimal fluctuational force [164].

We emphasize that the question of stability of a CA under small random perturbations is in itself an important unsolved problem in the theory of fluctuations $[92,93,94]$ and the difficulties in solving it are similar to those mentioned above. Thus it is unclear at first glance how an analogy between these two unsolved problems could be of any help. However, as already noted above, the new method for statistical analysis of fluctuational trajectories $[60,62,95,112]$ based on the prehistory probability distribution allows direct experimental insight into the almost deterministic dynamics of fluctuations in the limit of small noise intensity. Using this techique, it turns out to be possible to verify experimentally the existence of a unique solution, to identify the boundary condition on a CA, and to find an accurate approximation of the optimal control function.

Let us now formulate the problem of the energy-optimal steering of the motion from a chaotic attractor to the coexisting stable limit cycle for a simple model, a non-centrosymmetric Duffing oscillator. This is the model that, in the absence of fluctuations, has traditionally been considered in connection with a variety of problems in nonlinear optics [165]. Consider the motion of a periodically driven nonlinear oscillator under control

$$
\begin{aligned}
\dot{q}_{1} & =K_{1}(\mathbf{q}(t))=q_{2}, \\
\dot{q}_{2} & =K_{2}(\mathbf{q}(t))+u(t) \\
& =-2 \Gamma q_{2}-\omega_{0}^{2} q_{1}-\beta q_{1}^{2}-\gamma q_{1}^{3}+h \sin (\omega t)+u(t),
\end{aligned}
$$

Here $u(t)$ is the control function. It is a system where chaos can be observed at relatively small values $h \approx 0.1$ of the driving force amplitude and the chaotic attractor is a non-hyperbolic attractor or a quasiattractor [166].

We have considered the following energy-optimal control problem. The system (1.35) with unconstrained control function $u(t)$ is to be steered from a CA to a coexisting stable limit cycle (SC) in such a way that the "cost" functional $J$ is minimized, with $t_{1}$ unspecified

$$
R=\inf _{u \in U} \frac{1}{2} \int_{t_{0}}^{t_{1}} u^{2}(t) d t
$$


Here the control set $U$ consists of functions (control signals) able to move the system from the CA to the SC. The Pontryagin Hamiltonian (1.34) and the corresponding equations of motion take the form

$$
\begin{aligned}
& \dot{q}_{i}=\frac{\partial H_{c}}{\partial p_{i}}, \quad \dot{p}_{i}=-\frac{\partial H_{c}}{\partial q_{i}}, \quad i=\{1,2\}, \\
& H_{c}=1 / 2 p_{2}^{2}+p_{1} K_{1}+p_{2} K_{2} .
\end{aligned}
$$

Here it is assumed that the optimal control function $u(t)$ at each instant takes those values $u(t)=p_{2}$ that maximize $H_{c}$ over $U$.

We note that for $p_{1}=0$ and $p_{2}=0$ the dynamics of (1.37) reduces to the deterministic dynamics of the original system (1.35) in the absence of control $(u(t)=0)$. So we begin our analysis by considering some relevant properties of the deterministic dynamics of a periodically driven nonlinear oscillator.

The parameters of the system (1.35) were chosen such that the potential is monostable $\left(\beta^{2}<4 \gamma \omega_{0}^{2}\right)$, the dependence of the energy of oscillations on their frequency is nonmonotonic $\left(\frac{\beta^{2}}{\gamma \omega_{0}^{2}}>\frac{9}{10}\right)$, and the motion is underdamped $\Gamma \ll \omega \approx 2 \omega_{0}$.

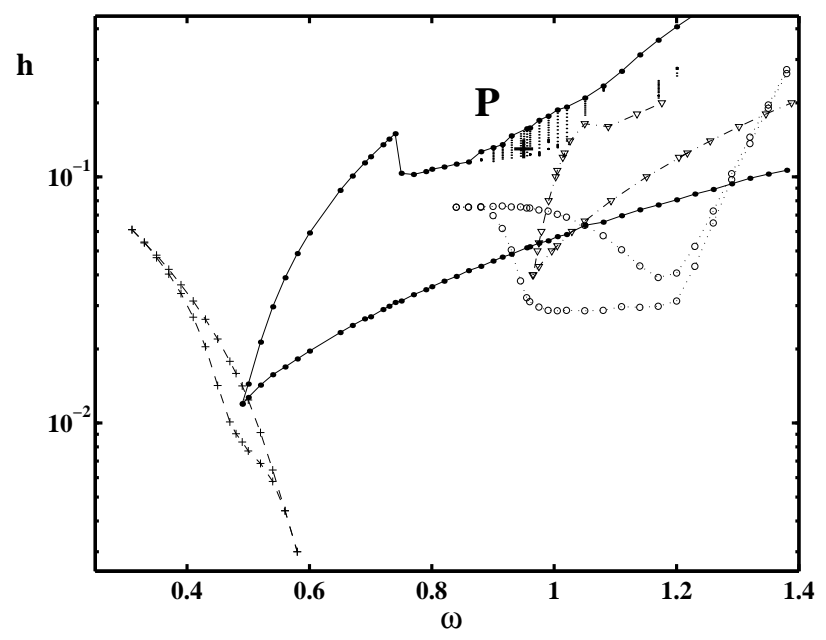

Fig. 1.13 Phase diagram of the system (1.35) on the $(\omega, h)$ plane obtained numerically for the parameter values $\Gamma=0.025, \omega_{0}=0.597, \beta=1, \gamma=1$. See text for a description of the symbols, the various lines are guide to the eye. The working point $P$, with $\omega_{f}=$ $0.95, h=0.13$, shown by a thick plus, was chosen to lie in the region of coexistence of the period 1 stable limit cycle and of the strange attractor [167].

A simplified parameter space diagram obtained numerically [167] is shown in Fig. 1.13. The dashed lines bound the region in which both the linear and nonlinear responses of period 1 coexist. The upper line marks the boundary of the linear response and the lower line marks that for the nonlinear responses. The boundaries 
of hysteresis for the period 1 resonance are shown by solid lines. The region in which linear response coexists with one or two nonlinear responses of period 2 is bounded by dotted lines. This region is similar to the one bounded by dashed lines. The region of coexistence of the two resonances of period 2 is bounded by the dashed-dotted line. Chaotic states are indicated by small dots. The chaotic state appears as the result

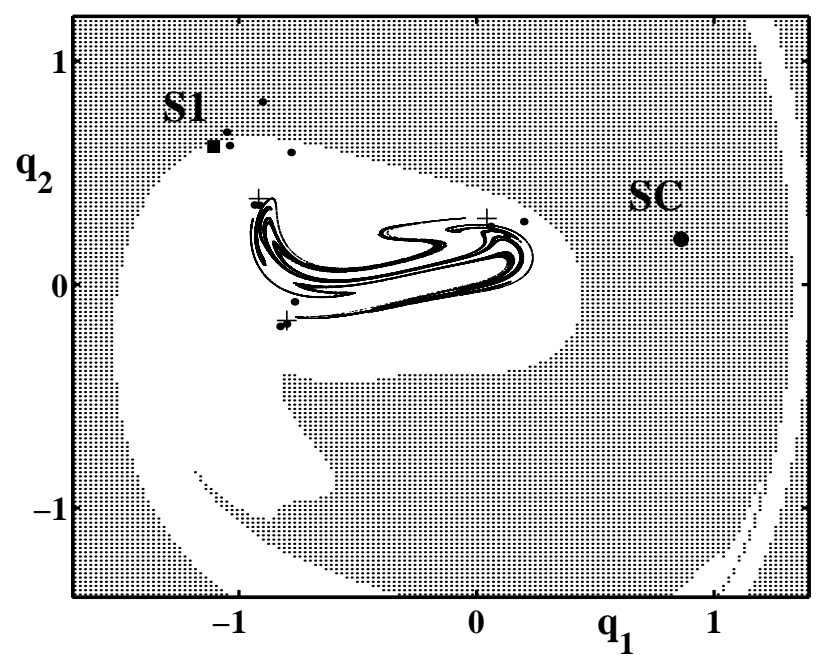

Fig. 1.14 The basins of attraction of the SC (shaded) and CA (white) for a Poincaré crosssection with $\omega_{f} t=0.6 \pi(\bmod 2 \pi), \omega_{f}=0.95$ in terms of $q_{1}$ at $q_{2}$. The boundary of the CA's basin of attraction, the saddle cycle of period 1, S1, is shown by the filled square. The saddle cycle of period 3, S3, is shown by pluses. The intersections of the actual escape trajectory with the Poincaré cross-section are indicated by the filled circles [168].

of period-doubling bifurcations, and thus corresponds to a non-hyperbolic attractor [166]. Its boundary of attraction $\partial \Omega$ is nonfractal and is formed by the unstable manifold of the saddle cycle of period 1 (S1).

For a given damping $(\Gamma=0.025)$ the amplitude and the frequency of the driving force were chosen so that the chaotic attractor coexists with the stable limit cycle (SC): $h=0.13, \omega_{f}=0.95$ (see Fig. 1.13).

The basins of attraction of the coexisting CA (strange attractor) and SC are shown in the Fig. 1.14 for the Poincaré cross-section $\omega_{f} t=0.6 \pi(\bmod 2 \pi)$ in the absence of noise [168]. The value of the maximal Lyapunov exponent for the CA is 0.0449 . The presence of the control function effectively doubles the dimension of the phase space (compare (1.35) and (1.37)) and changes its geometry near the non-hyperbolic attractor. In the extended phase space the attractor is connected to the basin of attraction of the stable limit cycle via an unstable invariant manifold. It is precisely the complexity of the structure of the phase space of an auxiliary Hamiltonian system (1.37) near the non-hyperbolic attractor that makes it difficult to solve the energyoptimal control problem. 
However, using the recently proposed $[60,62,95,112]$ method for experimental analysis of the Hamiltonian flow in an extended phase space of the fluctuating system, we can exploit an analogy between the Wentzel-Freidlin and Pontryagin Hamiltonians arising in the analysis of fluctuations, and the energy-optimal control problem in a nonlinear oscillator. To see how it can be done let us consider the fluctuational dynamics of a nonlinear oscillator (1.35).

Let us analyze the motion of an oscillator interacting with a thermal bath:

$$
\begin{aligned}
& \dot{q}_{1}=q_{2}, \\
& \dot{q}_{2}=-2 \Gamma q_{2}-\omega_{0}^{2} q_{1}-\beta q_{1}^{2}-\gamma q_{1}^{3}+h \sin (\omega t)+\xi(t), \\
& \langle\xi(t)\rangle=0, \quad\langle\xi(t) \xi(0)\rangle=D \delta(t)=4 \Gamma k T \delta(t) .
\end{aligned}
$$

In the zero-noise-intensity limit, a consistent theoretical development $[42,169]$ from the microscopic to the macroscopic equations of motion leads to descriptions of both its deterministic (dissipative) and fluctuational dynamics within the framework of Hamiltonian formalism [57]. The comparison of the Hamiltonian approach to large fluctuations, described in Sec. 1.3, and the approach to optimal control problem shows that, both on physical grounds and rigorously, the Wentzel-Freidlin Hamiltonian [57] (1.37) is equivalent to the Pontryagin Hamiltonian (1.34) [163] and the corresponding optimal control function is equivalent to the optimal fluctuational force. The analogy between the two problems opens up the possibility of a direct experimental insight into the geometry of the phase space of system (1.34) using a statistical analysis of the fluctuational trajectories in this system when a control function $u(t)$ is substituted for the random function $\xi(t)$. In particular the optimal control signal $\bar{u}(t)$ can be identified with the optimal fluctuational force which drives the system from the chaotic attractor to the stable limit cycle [164]. We note that both $\bar{u}(t)$ and the optimal force are related to $p_{2}$ in (1.37) (see e.g. in [143]).

We therefore suggest that the optimal control function $\bar{u}(t)$ can be found experimentally by measurement of the optimal fluctuational force $[95,112]$.

This interrelationship is intuitively clear because, in thermal equilibrium $(D=$ $4 \Gamma k_{B} T$ ), the probability of fluctuations is determined by the minimum work of the external source needed to produce the given change in the thermodynamic quantities $\rho \propto \exp \left(-R_{\min } / k_{B} T\right)$ [9]. We emphasize that the analysis presented above draws an analogy between two quite distinct and separate problems: the deterministic energy-optimal control problem, and the problem of the stability of the system in the presence of small random perturbations. Very similar conclusions can be drawn using a more general formulation of the stochastic optimal control problem (see e.g. [164]).

1.5.1.2 Statistical analysis of fluctuational trajectories A statistical analysis of the fluctuational trajectories is based on the measurements of the prehistory probability distribution [60] $p_{h}\left(q, t ; q_{f}, t_{f}\right)$ (see Sec. 1.3). By investigating the prehistory probability distribution experimentally, one can establish the area of phase space within which optimal paths are well defined, i.e. where the tube of fluctuational paths around an optimal path is narrow. The prehistory distribution thus provides information about both the optimal path and the probability that it will be followed. 
In practice the method essentially reduces to continuously following the dynamics of the system and constructing the distribution of all realizations of the fluctuational trajectories that transfer it from a state of equilibrium to a prescribed remote state.

To find the optimal control function $\bar{u}(t)$ we performed digital simulations of (1.38) using the Heun algorithm, with particular care given to the random number generator (see $[142,170]$ ), because simulation times necessarily grow exponentially as $D \rightarrow 0$. We have also carried out a complementary analog electronic modeling [112] of (1.38). We drive it with zero-mean quasi-white Gaussian noises from a noise generator, digitize the response $q_{1}(t), q_{2}(t), \xi(t)$, and analyze it with a digital data processor. In both analog and digital simulations, trajectories moving the system from the chaotic attractor to the stable limit cycle were collected, and the corresponding distributions of the escape trajectories were built and analyzed. Qualitatively similar results were obtained but, because precision is of particular importance here, most of the data reported below are those from the digital simulations.

For the technique to be applicable, a solution of (1.37) moving the system from the CA to $\partial \Omega$ must exist, and one has to be able to identify the boundary conditions for this solution on the CA.

In the presence of weak noise there is a finite probability of noise-induced transitions between the chaotic attractor and a stable limit cycle. In Fig. 1.14 the filled circles show the intersections of one of the real escape trajectories with the given Poincaré section. The following intuitive escape scenario can be expected in the Hamiltonian formalism. Let us consider first the escape of the system from the basin of attraction of a stable limit cycle that is bounded by an saddle cycle. In general, escape occurs along a single optimal trajectory $q_{o p t}(t)$ connecting the two limit cycles.

The trajectory $q_{\text {opt }}(t)$ is determined by minimizing $S$ in (1.20) on the set of all classical deterministic trajectories determined by the Hamiltonian $H$ (1.37), that start on a stable limit cycle as $t \rightarrow-\infty$ and terminate at an saddle cycle as $t \rightarrow \infty$. That is, $q_{o p t}(t)$ is a heteroclinic trajectory of the system (1.37) with minimum action, where the minimum is understood in the sense indicated, and the escape probability assumes the form $P \approx \exp (-S / D)$. We note that the existence of optimal escape trajectories and the validity of the Hamiltonian formalism have been confirmed experimentally for a number of nonchaotic systems (see Refs. [62, 95, 112, 131, 171] and references therein).

If the noise is weak, then the probability $P \approx \exp (-S / D)$ to escape along the optimal trajectory is exponentially small, but it is exponentially greater than the escape probability along any other trajectory, including along other heteroclinic trajectories of the system (1.37).

Since the basin of attraction of the CA is bounded by an saddle cycle S1, the situation near S1 remains qualitatively the same and the escape trajectory remains unique in this region. However, the situation is different near the chaotic attractor. In this region it is virtually impossible to analyze the Hamiltonian flux of the additional system (1.37), and no predictions have been made about the character of the distribution of the optimal trajectories near the CA. The simplest scenario is that an 
optimal trajectory approaching the boundary of a chaotic attractor is smeared into a "cometary tail" and is lost, merging with the boundary of the attractor.

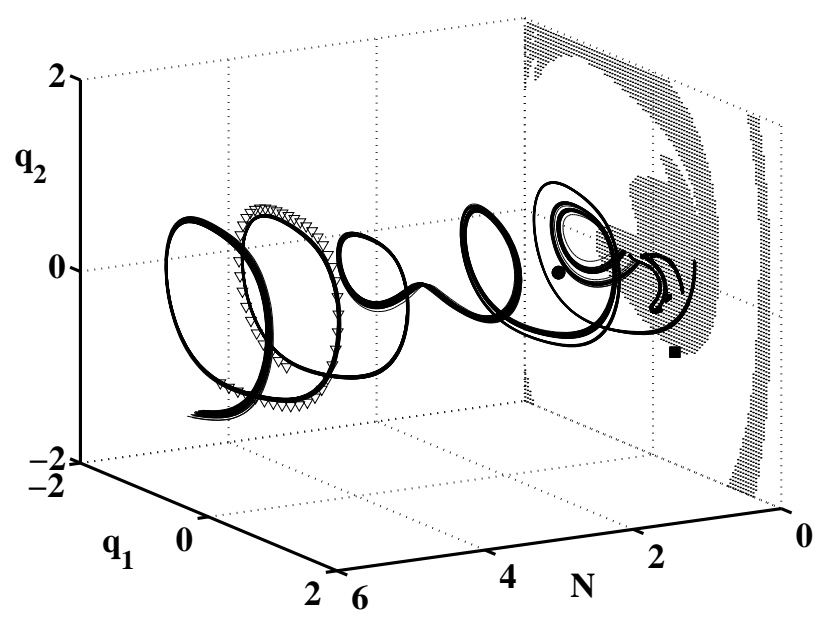

Fig. 1.15 Escape trajectories found [172] in the analogue simulations for the parameters $h=0.19, \omega_{f} \approx 1.045, \omega_{0} \approx 0.597, D \approx 0.0005$ are shown in comparison with the Poincaré cross-section of a quasi-attractor and its basins of attraction for $\omega_{f} t=0$.

However, statistical analysis of real fluctuation-induced escape trajectories gives a more detailed picture of the noise-induced escape from a chaotic attractor. Several thousand real escape trajectories of the system (1.38) from the basin of attraction of a CA in various operating regimes were investigated [172]. The typical situation as measured in analog simulations is displayed in Fig. 1.15 for system parameters close to the point $\mathrm{P}$ in Fig. 1.13 and a noise intensity $D \approx 0.0005$. The figure shows 65 measured fluctuational escape trajectories. All the trajectories have been shifted in time so that the characteristic regions of the trajectories corresponding to the transition from chaotic to regular motion coincide with each other.

It is evident that all real trajectories pass through the close neighborhood of some optimal trajectory in a tube with a radius $\propto \sqrt{D}$. Therefore it is possible to determine the optimal escape paths by simple averaging performed separately for each group of trajectories. The number of different optimal escape paths obtained for the transition $\mathrm{CA} \rightarrow \mathrm{S} 3$ depends on the choice of the working point. From one to three distinct optimal escape paths for operation in various regimes were observed experimentally. The escape probabilities along different paths are different, and, as the noise intensity is reduced, one of the escape paths becomes exponentially more probable then the others. In what follows we concentrate on the properties of this most probable escape path.

To find the boundary conditions on the $\mathrm{CA}$, we analyze the prehistory probability distribution $p_{h}\left(q, t ; q_{f}, t_{f}\right)$ of the escape trajectories. The corresponding distribution 
is shown in the Fig. 1.16. It can be inferred by the inspection of how the ridge of the most probable escape path merges the CA that most of the escape trajectories pass close to the saddle cycle of the period 5 embedded into the CA.

This hypothesis can be elaborated further using a statistical analysis of the trajectories arriving to a small tube around $\mathrm{S} 3$ for the nose intensity reduced by a few orders of magnitude up to $D=1.5 \times 10^{-6}$, see Fig. 1.17 [172]. The statistical analysis

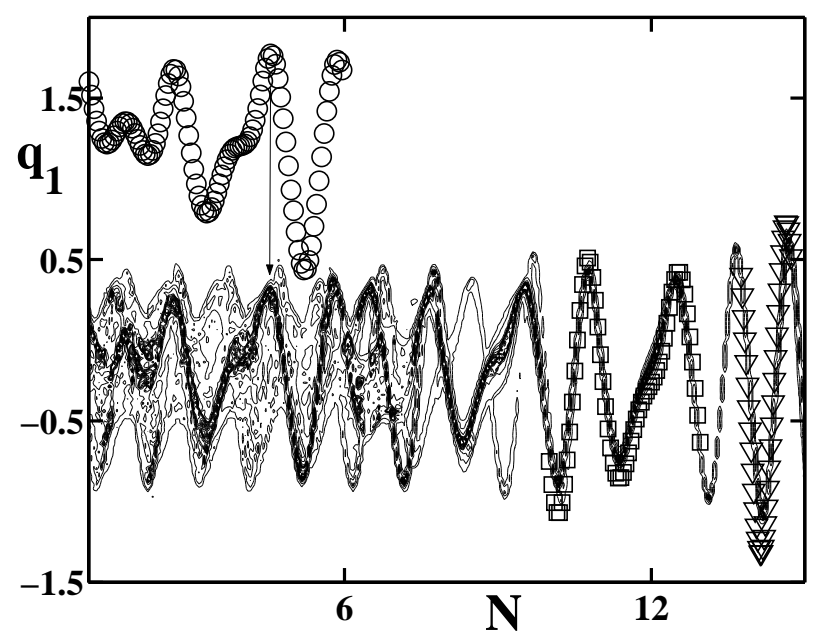

Fig. 1.16 The prehistory probability distribution of the escape trajectories for the parameters as in Fig. 1.13. The circles, squares and triangles show single periods of the saddle cycles of period 5 (S5), 3 (S3), and 1 (S1), respectively [172].

of the escape trajectories described in the subsection reveals that the energetically favorable way to move the system from the CA to the stable limit cycle starts at the saddle cycle of period 5 (S5) embedded in the CA, passes through saddle cycle S3 and finishes at the saddle cycle $\mathrm{S} 1$ at the boundary of the basin of attraction of the CA. Subsequent motion of the system towards the stable limit cycle does not require external action.

To find an approximation to the optimal control function we collect all successful realisations $\left(q_{1}^{e s c}(t), q_{2}^{e s c}(t), \xi^{e s c}(t)\right)$ that move it from $\mathrm{S} 5$ to $\partial \Omega$. An approximate solution $\tilde{u}(t)$ is then found as an ensemble average over the corresponding realisations of the random force $\left\langle\xi^{e s c}(t)\right\rangle$ (the exact solution is $\bar{u}(t)=\lim _{D \rightarrow 0} \tilde{u}(t)$ ). The results of this procedure are shown in the upper trace of Fig. 1.18. To remove the irrelevant high-frequency component left after averaging, we filtered through a zero-phase low-pass filter with frequency cut-off $\omega_{c}=1.9$.

It can be seen from the figure that the optimal force switches on at the moment when the system leaves S5 along its unstable manifold. The optimal force returns to zero when the system reaches the saddle cycle $\mathrm{S} 1$. 


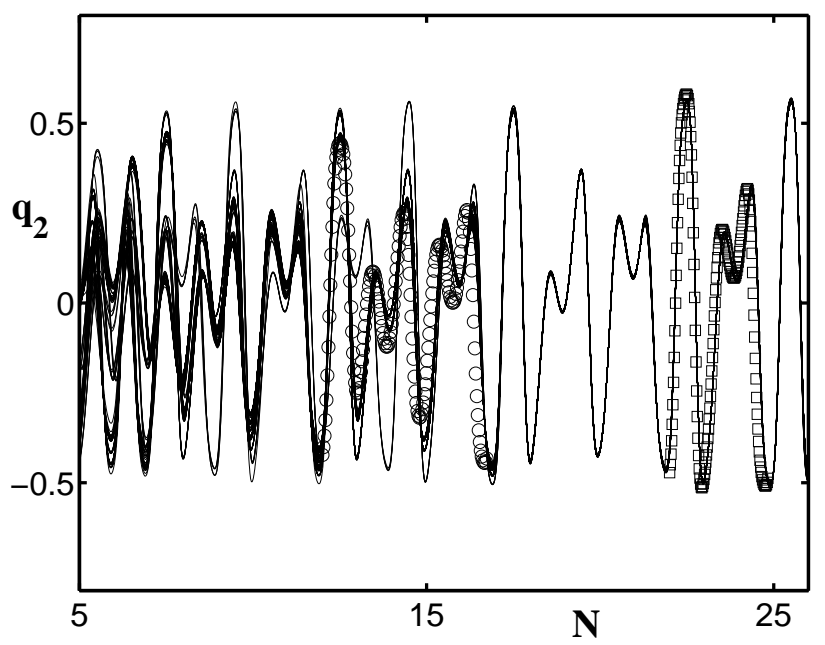

Fig. 1.17 Escape trajectories for the parameters as in Fig. 1.16. The squares and circles show one period of the saddle cycle S3 and one period of S5, respectively.

Thus we conclude that the solution $\tilde{u}(t)$ and the corresponding boundary conditions can be found using our new experimental method. Moreover the problem of escape from the CA of a periodically driven nonlinear oscillator can be essentially reduced to the analysis of a transition between three saddle cycles $\mathrm{S} 5 \rightarrow \mathrm{S} 3 \rightarrow \mathrm{S} 1$. We note that the latter result is in qualitative agreement with the well known statement that unstable cycles provide detailed invariant characterizations for dynamical systems of low intrinsic dimension (see e.g. [173, 174, 175]).

This result opens up the possibility of the numerical solution of the corresponding boundary value problem for the energy-optimal control formulated above.

It can be shown [172] that the average time for the system to approach S5 is much smaller then the average escape time and thus the optimal escape paths found from the statistical analysis of the escape trajectories is independent on the initial conditions on the attractor and provides an approximation to the global minimum of the corresponding deterministic control problem.

1.5.1.3 Numerical solution of the boundary value problem In principle, it is possible to find the optimal path by direct solution of the Pontryagin Hamiltonian (1.37), with appropriate boundary conditions. We must stress that even for this relatively simple system, the solution is a formidable, and almost impossible, task. First of all, in general one has no insight into the appropriate boundary conditions, in particular into those at the starting time (which belong to the strange attractor). But even if the boundaries were known, in practice the determination of the optimal path is impossible: the functional $R$ of Eq. (1.36) has so many local minima, that it proved impractical to attempt a (general) search for the optimal path. 


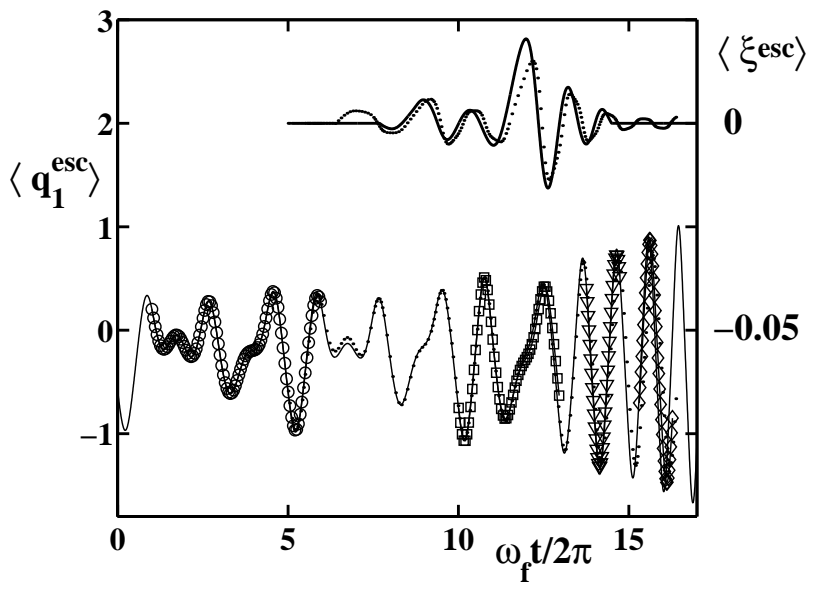

Fig. 1.18 The most probable escape path (bottom solid curve) from S5 to the S1, found in the numerical simulations. The stable limit cycle is shown by rombs, see Fig. 1.16 for other symbols. Parameters were $h=0.13, \omega_{f}=0.95, \omega_{0} \approx 0.597, D=0.0005$. Top: optimal force (solid line) corresponding to the optimal path after filtration[168], and optimal force from numerical solution of the boundary value problem (dots).

However, once the fluctuational trajectories were available, we did indeed manage to find the optimal path using by direct solution of the Pontryagin Hamiltonian. The idea is to study the escape scenario which emerges from the fluctuational trajectories: as we mentioned, the escape takes place through S3, S5 and S1. We then built an initial trial function, taking a linear combination of the structures involved in the escape. The combination was such that at short times the trial function coincided with S5, while at large times it coincided with S1. At intermediate times, we had a mixture of S5 and S1 in the initial trial function, but no S3. Using a well known algorithm for two points boundary conditions (TWPBVP, obtained via netlib [176], see [177] for details), we then relaxed this trial function to find the optimal path, defined as the path which minimises the functional 1.36. It is striking that the relaxational optimal path that we found does go through S3, in good agreement with what was observed for the optimal path obtained via the fluctuational trajectories (for details, see [172]).

1.5.1.4 The energy-optimal migration control of a chaotic oscillator Here we examine the control of migration in a periodically driven nonlinear oscillator. Our aim is to demonstrate that application of the approximate solution found from the statistical analysis of fluctuational trajectories optimizes (minimizes) the energy of the control function. We compare the performance of some known adaptive control algorithms to that of the control function found through our analysis.

To verify that the optimal force $\tilde{u}(t)$ found in the experiment does minimize the energy of the control function steering the system (1.35) from the CA to the S1, we set it to arbitrary initial conditions in the basin of attraction of CA and let it evolve 
deterministically until it passed through the initial part of the unstable manifold of S5. At this moment the deterministic control function was switched on. For small variations in the shape of the control function and/or initial conditions, the amplitude of the control function was set to the threshold of the switching for the system from chaotic motion to regular motion on the stable limit cycle. It was found that the
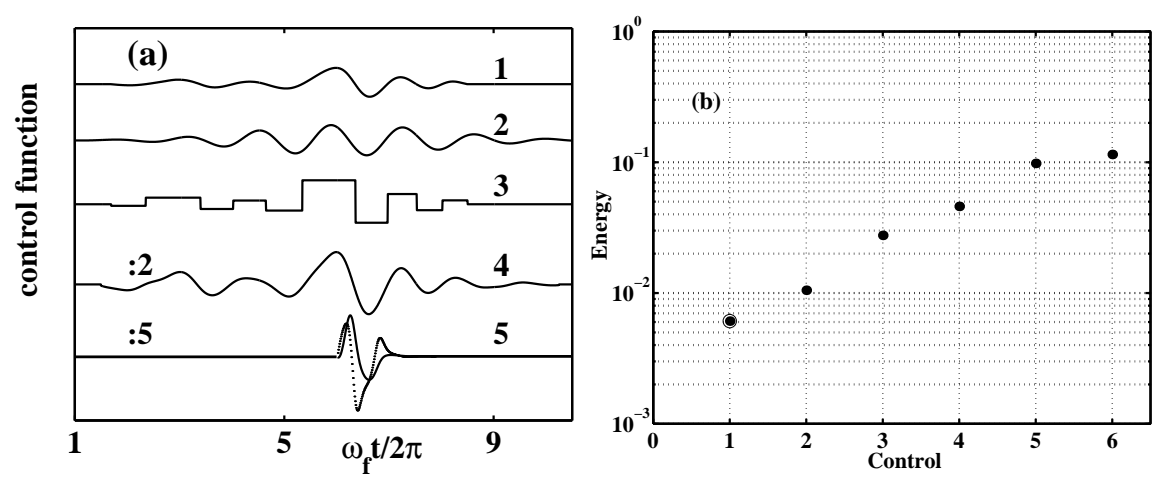

Fig. 1.19 (a) The shapes of the control functions (not drawn to scale) used in the numerical experiment: 1 - optimal force found from the statistical analysis of the fluctuational escape trajectories; 2 - approximation of the optimal force by the $u(t)=a_{1} \sin \left(a_{2} t\right) \exp (-(t-$ $\left.\left.a_{3}\right)^{2} a_{4}\right)$ where $a_{i}$ are constants; 3 - approximation of the optimal force by the rectangular pulses; 4 - arbitrary perturbation of the optimal force with a low-frequency perturbation; 5 - control functions produced by the OPCL algorithm; 6 - control function for the adaptive control. (b) Energies of the control functions shown in (a) [168].

system is very sensitive to variation of both the shape of the control function and the initial conditions. It was also demonstrated that any deviation from the shape of $\tilde{u}(t)$ or from the initial conditions found in the experiment leads to a substantial increase in the energy of the control function required to steer system from a CA to S1. Some experimental results are shown in Fig. 1.19. Thus it can be seen that the energy of the control function is approximately twice larger if the optimal force is approximated by the $\sin$ function modulated by the Gaussian $u(t)=a_{1} \sin \left(a_{2} t\right) \exp \left(-\left(t-a_{3}\right)^{2} a_{4}\right)$ and it is $\sim 4$ and 20 times larger if the optimal force is approximated by rectangular pulses or perturbed with arbitrary low-frequency perturbations respectivelly.

We have also performed experiments using an open-plus-closed-loop control technique [155] and adaptive control algorithm [156] to steer the system from the CA to the $\mathrm{S} 1$. The equations of motion are taken in the form

$$
\begin{aligned}
& \dot{q}_{1}=q_{2}+F_{1}(q, g, t), \\
& \dot{q}_{2}=-2 \Gamma q_{2}-\omega_{0}^{2} q_{1}-\beta q_{1}^{2}-\gamma q_{1}^{3}-f \cos (\omega t) \\
& +F_{2}(q, g, t), \quad q=q_{1}, q_{2}, \quad g=g_{1}, g_{2}
\end{aligned}
$$


Here $F(q, g, t)$ is the control function in the form

$$
F(q, g, t)=(\dot{g}-K(g))+S(t)\left(K^{\prime}(g)-A\right)(g(t)-q(t)) .
$$

We will be interested in the situation when the "goal dynamics" $g(t)$ is a solution of (1.35) with $u(t)=0$, i.e. $\dot{g}=K(g)$. Namely $g(t)$ describes the stable limit cycle of period $1 \mathrm{SC}$ coexisting with the CA. Thus the first term in (1.40) vanishes. And $F(q, g, t)$ takes the following explicit form

$$
F_{i}(q, g, t)=S(t) \sum_{j=1,2}\left(K_{i j}-a_{i j}\right)\left(g_{j}-q_{j}\right)
$$

Here $i=1,2$ and $K_{i j}=\partial K_{i} / \partial q_{j}$. We have considered only the case $a_{i j}=$ $-\left|a_{i} j\right| \delta_{i j}$ and $S(t)=1-\exp (-\lambda t)$ as it was suggested in [156]. Parameters $|a|$ and $\lambda$ were varied to optimize the energy of the control function.

The energy of the control functions obtained by these methods varies from 0.14 to 0.6 and thus it is more then one order of magnitude larger then the energy of the optimal control function $\tilde{u}(t)$ found by our new technique. (see Fig. 1.19). Similar results were obtained using the algorithm for adaptive chaos control [150] for the migration of the nonlinear oscillator from the CA to SC (see Fig. 1.19).

We note that neither the OPCL nor the adaptive control algorithms were devised to optimize the energy of the control, but rather the recovery time. It is clear that these methods are insensitive to the initial conditions at the CA. The shapes of the control functions are, to a large extent, also prescribed by the algorithms and are not optimized. In this sense the high energy of the control functions is not a surprise: the results presented serve the purpose to illustrate the main point, i.e. the sensitivity of the optimal control to the shape of the control function and to the initial conditions, discussed above.

\subsubsection{Fluctuational escape from a quasi-hyperbolic attractor}

We now consider, for comparison, fluctuational escape from the Lorenz attractor, which, for a certain range of parameters, is a quasi-hyperbolic attractor consisting of unstable sets only [160]:

$$
\begin{array}{rll}
\dot{q_{1}} & = & \sigma\left(q_{2}-q_{1}\right), \\
\dot{q_{2}} & = & r q_{1}-q_{2}-q_{1} q_{3}, \\
\dot{q_{3}} & = & q_{1} q_{2}-b q_{3}+\xi(t), \\
\langle\xi(t)\rangle=0, & & \langle\xi(t) \xi(0)\rangle=D \delta(t)
\end{array}
$$

In the absence of noise, the system [178] describes the generation of a single-mode laser field interacting with a homogeneously broadened two-level medium [179]. The variables and parameters of the Lorenz system can be interpreted in terms of a laser system as: $q_{1}$ is the normalized electric field amplitude, $q_{2}$ the normalized polarization, $q_{3}$ the normalized inversion, $\sigma=k / \gamma_{1}, r=\Lambda+1, b=\gamma_{2} / \gamma_{1}$, with 
$k$ the cavity decay rate of the field in the cavity, $\gamma_{1}$ and $\gamma_{2}$ the relaxation constants of the inversion and polarization, and $\Lambda$ the pump parameter. Far-infrared lasers have been proposed as an example of a realization of the Lorenz system [161]. A detailed comparison of the dynamics of the system (1.42) and a far-infrared laser, plus a discussing the validity of the Lorenz system as laser model, can be found in [162].

The Lorenz equations have a simple structure and contain two nonlinear terms only. Let us briefly consider the main bifurcations in the system (1.42) (a more detailed analysis can be found in [180]). We fix the parameters $\sigma=10, b=8 / 3$ and vary the parameter $r$ : in this case two global bifurcations take place (see the bifurcation diagram in Fig. 1.20). For $r=1$, a supercritical pitchfork bifurcation happens: the stationary state at the origin $O \equiv(0,0,0)$ becomes a saddle state and two new stationary states appear: $P_{1} \equiv(\sqrt{b(r-1)}, \sqrt{b(r-1)}, r-1)$ and $P_{2} \equiv(-\sqrt{b(r-1)},-\sqrt{b(r-1)}, r-1)$. In the system phase space there are two stable points $P_{1}$ and $P_{2}$, a saddle point $O$ at the origin, and their one-dimensional (separatrixes) and two-dimensional manifolds.

The second bifurcation occurs at

$$
r=\frac{\sigma(\sigma+b+3)}{(\sigma-b-1)} \approx 24.74 \ldots
$$

and it is a subcritical Hopf bifurcation, when states $P_{1}$ and $P_{2}$ loss their stability and in the phase space there is the unique chaotic quasi-hyperbolic attractor.

There are also two local bifurcations. The first one takes place for $r \approx 13.926 \ldots$, when a homoclinic tangency of separatrixes of the origin $O$ occurs (it is not shown in Fig. 1.20) and a hyperbolic set appears, which consists of a infinite number of saddle cycles. Beside the hyperbolic set, there are two saddle cycles $L_{1}$ and $L_{2}$ around the stable states $P_{1}$ and $P_{2}$. The separatrixes of the origin $O$ withdraw to the saddle cycles $L_{1}$ and $L_{2}$, and the attractors of system are the states $P_{1}$ and $P_{2}$. The second local bifurcation is observed for $r \approx 24.06$. The separatrixes do not withdraw to the saddle cycles $L_{1}$ and $L_{2}$. As a result, in the phase space of the system a stable quasi-hyperbolic state appears - the Lorenz attractor. The chaotic Lorenz attractor includes separatrices, the saddle point $O$ and a hyperbolic set, which appears as a result of homoclinic tangency of separatrices. The presence of the saddle point in the chaotic attractor defines the prefix "quasi" in the definition of the chaotic attractor as quasi-hyperbolic [160]. The states $P_{1}, P_{2}$ remain stable. Thus, in the range $r \in[24.06: 24.74]$ the co-existence of the chaotic attractor and two stable point-attractors is observed in the phase space of the Lorenz system. Let us fix the parameter $r=24.08$ in this range and consider the noise-induced escape for the chaotic attractor to the basins of attraction of the stable points. Note that in [64] the invariant measure of the noisy Lorenz attractor was found within a Hamiltonian formalism, but large deviations from a chaotic attractor were not considered.

First, we examine [181] the structure of the system phase space for chosen parameters $\sigma=10, b=8 / 3, r=24.08$ (Fig. 1.21). 


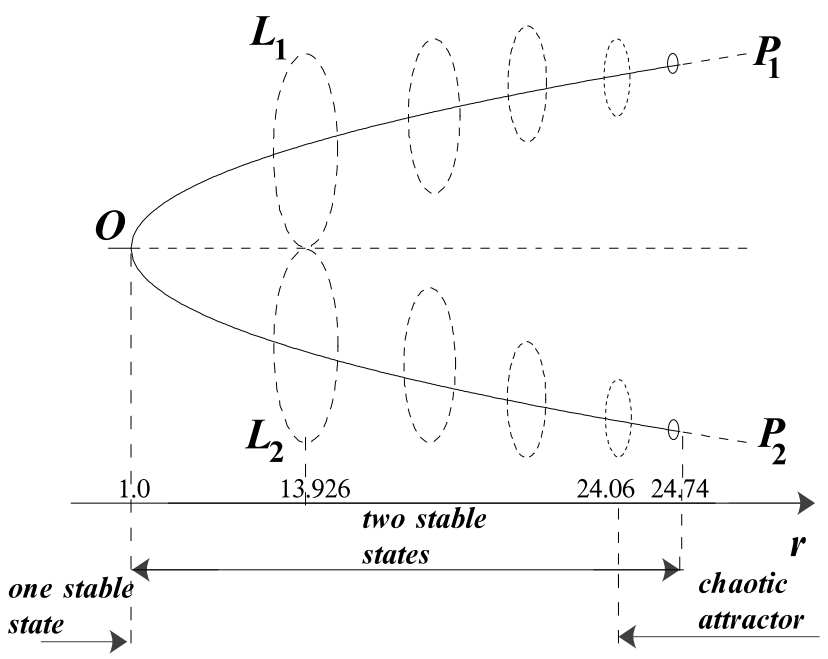

Fig. 1.20 The bifurcation diagram of the lorenz system for fixed $\sigma=10, b=8 / 3$. The unstable and stable sets are shown by dashed and solid lines respectively.

The saddle cycles $L_{1}$ and $L_{2}$ surround the stable states $P_{1}$ and $P_{2}$ and they are located at the intersection of the unstable $W^{u}$ and stable $W^{s}$ manifolds. The unstable manifold goes to the stable state $P_{1}$ from one side and to the chaotic attractor from the other side. The stable manifold $W^{s}$ forms a tube in the vicinity of the stable state [182]. The saddle cycles $L_{1}$ and $L_{2}$ have the multipliers $(1.0000,1.0280,0.0001)$, and therefore trajectories will go slowly away along the unstable manifold and they will approach quickly along the stable manifold.

For simplicity we add noise in the form of a white noise $\xi(t)$ to the third equation of system (1.42), preserving the original system symmetry.

Like the escape from a non-hyperbolic attractor, there is no theoretical prediction about the process of fluctuational escape from the Lorenz attractor. But the process is readily studied via numerical simulation and via analysis of the prehistory probability distribution built using the fluctuational escape trajectories. For definiteness, we examine escape to the stable point $P_{1}$. The averaged escape trajectory and the corresponding averaged fluctuational force obtained in this way are shown in Fig. 1.22. We have found that the escape occurs via the following scenario. The escape trajectory starts from the stable manifold of the saddle point $O$. Under the action of a fluctuation, an escape trajectory tends to point $O$ along the two-dimensional stable manifold. Then, without reaching the saddle point $O$, the trajectory departs from it again, following a path close to the separatrix $\Gamma_{2}$, and falling into the neighborhood of the saddle cycle $L_{1}$. In the absence of an external force, the trajectory goes away from the cycle $L_{1}$, slowly untwisting. The fluctuational force induces a crossing through the saddle cycle, and the trajectory then relaxes to the stable point $P_{1}$. We can thus split the escape process into two parts: fluctuational and relaxational. In practice all the fluctuational part belongs to the Lorenz attractor, and itself consists of 


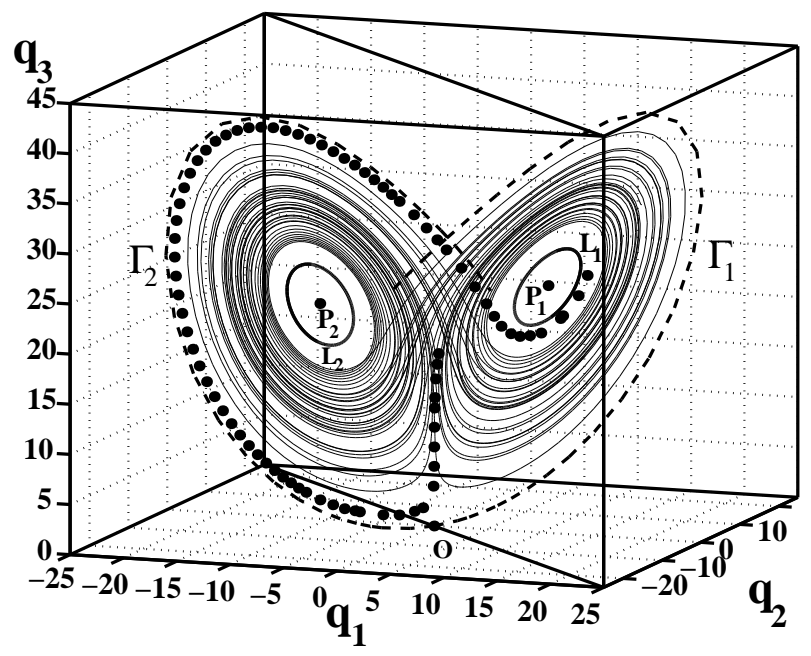

Fig. 1.21 Structure of the phase space of the Lorenz system. An escape trajectory measured by numerical simulation is indicated by the filled circles. The trajectory of the Lorenz attractor is shown by a thin line, the separatrixes $\Gamma_{1}$ and $\Gamma_{2}$ by dashed lines [181]

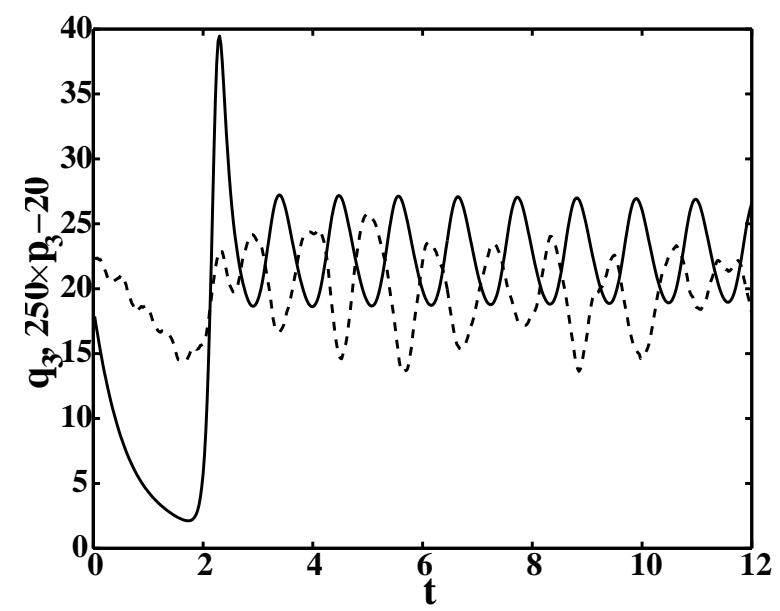

Fig. 1.22 The averaged escape trajectory (solid line) and the averaged fluctuational force (dashed line) during escape from the Lorenz attractor [181].

two stages: at first, the fluctuational force throws the trajectory as close as possible to the cycle $L_{1}$; then, the trajectory crosses this cycle under the action of fluctuations. The first stage is defined by the stable and unstable manifolds of the saddle point $O$, and the time-dependence of the fluctuational force is similar to that of the coordinate $q_{3}$ (Fig. 1.22). During the second stage, the fluctuations have a component which 
oscillates in anti-phase to the coordinate $q_{3}$. Because the trajectory of the noise-free system departs from the cycle $L_{1}$ very slowly, the fluctuational force inducing the crossing through the cycle may start to act at any time during a long interval. For this reason the averaged fluctuational force itself consists of a long oscillating function.

It is clear that all of the escape trajectory from the Lorenz attractor lies on the attractor itself. The role of the fluctuations is, first, to bring the trajectory to a seldomvisited area in the neighborhood of the saddle cycle $L_{1}$, and then to induce a crossing of the cycle $L_{1}$. So we may conclude that the role of the fluctuations is different in this case, and the possibility of applying the Hamiltonian formalism will require a more detailed analysis of the crossing process.

Thus, we have found that the mechanisms of escape from a non-hyperbolic attractor and a quasi-hyperbolic (Lorenz) attractor are quite different, and that the prehistory of the escape trajectories reflects the different structure of their chaotic attractors. The escape process for the non-hyperbolic attractor is realized via several steps, which include transitions between low-period saddle-cycles co-existing in the system phase space. The escape from the Lorenz attractor consist of two qualitatively different stages: the first is defined by the stable and unstable manifolds of the saddle center point, and lies on the attractor; the second is the escape itself, crossing the saddle boundary cycle surrounding the stable point attractor. Finally, we should like to point out that our main results were obtained via an experimental definition of optimal paths, confirming our experimental approach as a powerful instrument for investigating noise-induced escape from complex attractors.

\subsection{CONCLUSIONS}

The recent rapid advances in the understanding of fluctuating nonlinear systems, including optical systems, have come about in large part through the mutually supportive relationship between analytic calculation and analog and digital simulations. This has been especially true of problems involving large fluctuations, where use of simulations, coupled with the introduction of the prehistory probability distribution, have set the area on an experimental basis for the first time, and helped to stimulate new advances in the theory. These have included the logarithmic susceptibility, described above, which promises to do for optimal paths what the conventional linear susceptibility has done for linear response theory. The theory of the logarithmic susceptibility in turn has been tested, and its limits of applicability explored, through simulations. And the same is true of recent developments in understanding Kramers' problem on short timescales. Studies of the fluctuational escape from chaotic attractors, of which two examples is described above, are entirely simulation-led at present. But the results of the analogue and digital experiments have already provided strong guidance for future developments in the theory. It seems certain that the close symbiotic relationship between simulation and theory in fluctuational dynamics will continue, and that the emergence of many new results and phenomena may be anticipated over the next few years. 


\section{Acknowledgements}

We warmly acknowledge the assistance and support of our many collaborators in the research reviewed in this chapter including, especially, I.Kh. Kaufman, V.N. Smelyanskiy, S.M. Soskin, N.D. Stein and N.G. Stocks. But, most of all, we would like to acknowledge our continuing indebtedness to M.I. Dykman whose insights and ideas now permeate the entire field of fluctuational dynamics, and with whom it has been such a privilege and pleasure to collaborate over many years. The research has been supported in part by the Engineering and Physical Sciences Research Council (UK), INTAS, the EC, the Royal Society of London, and by Award No. REC-006 of the U.S. Civilian Research \& Development Foundation for the Independent States of the Former Soviet Union (CRDF).

\section{REFERENCES}

1. M. I. Dykman, D. G. Luchinsky, R. Mannella, P. V. E. McClintock, N. D. Stein, and N. G. Stocks, Adv. Chem. Phys. LXXXV, 265 (1994)

2. A. Einstein, Ann. Phys. 17, 549 (1905).

3. M. von Smoluchowski, Bull. Intern. Acad. D. Sc. Cracovie (A) 418 (1913).

4. P. Langevin, Comptes Rendus 146, 530 (1908).

5. W. H. Louisell, Radiation and Noise in Quantum Electronics, McGraw-Hill, New York, 1964.

6. M. O. Scully and W. E. Lamb, Jr., Phys. Rev. 159, 208 (1967).

7. M. Lax, Fluctuation and Coherence Phenomena in Classical and Quantum Physics, Gordon and Breach, New York, 1968.

8. H. Haken in S. Flugge, ed., Encyclopaedia of Physics, Springer, Berlin, 1970, vol.XXV/2c.

9. L. D. Landau and E. M. Lifshitz, Statistical Physics, 3rd Edn, part 1, Pergamon, New York, 1980.

10. R. Short, L. Mandel, and R. Roy, Phys. Rev. Lett. 49, 647 (1982).

11. See, for example, OSA Proceedings on Nonlinear Dynamics in Optical Systems, N. B. Abraham, E. Garmire and P. Mandel, eds., Optical Society of America, Washington, 1991; and subsequent volumes in this proceedings series.

12. R. Benzi, A. Sutera, and A. Vulpiani, J. Phys. A 14, L453 (1981); R. Benzi, G. Parisi, A. Sutera, and A. Vulpiani, Tellus 34, 10 (1982).

13. C. Nicolis, Tellus 34, 1 (1982). 
14. M. I. Dykman, D. G. Luchinsky, R. Mannella, P. V. E. McClintock, N. D. Stein, and N. G. Stocks, J. Stat. Phys. 70, 479 (1993).

15. M. I. Dykman, D. G. Luchinsky, R. Mannella, P. V. E. McClintock, N. D. Stein, and N. G. Stocks, Nuovo Cimento D 17, 661 (1995).

16. L. Gammaitoni, P. Hänggi, P. Jung, and F. Marchesoni, Rev. Mod. Phys. 70, 223 (1998).

17. D. G. Luchinsky, R. Mannella, P. V. E. McClintock, and N. G. Stocks, IEEE Trans. on Circuits and Systems II: Analog and Digital Signal Processing 46, 1205 (1999).

18. D. G. Luchinsky, R. Mannella, P. V. E. McClintock, and N. G. Stocks, IEEE Trans.,on Circuits and Systems II: Analog and Digital Signal Processing 46, 1215 (1999).

19. B. McNamara, K. Wiensenfeld, and R. Roy, Phys. Rev. Lett. 60, 2626 (1988).

20. G. Vemuri and R. Roy, Phys. Rev. A 39, 4668 (1989).

21. A. Fioretti, L. Guidoni, R. Mannella, and E. Arimondo, J. Stat. Phys. 70, 403 (1993).

22. J. M. Iannelli, A. Yariv, T. Chen, and Y. Zhuang, Appl. Phys. Lett. 65, 1983 (1994).

23. M. I. Dykman, A. L. Velikovich, G. P. Golubev, D. G. Luchinskii, and S. V. Tsuprikov, Soviet Phys. JETP Lett. 53, 193 (1991).

24. J. Grohs, S. Apanasevich, P. Jung, H. Issler, D. Burak, and C. Klingshirn, Phys. Rev. A 49, 2199 (1994).

25. B. Jost, and B. Saleh, Opt. Lett. 21, 287 (1996).

26. F. Vaudelle, J. Gazengel, G. Rivoire, X. Godivier, and F. Chapeau-Blondeau, J. Opt. Soc. Am. B 15, 2674 (1998).

27. A. Simon, and A. Libchaber, Phys. Rev. Lett., 68, 3375 (1992).

28. M. I. Dykman, G. P. Golubev, D. G. Luchinsky, P. V. E. McClintock, N. D. Stein, and N. G. Stocks, Phys. Rev. E 49, 1935 (1994).

29. M. I. Dykman, G. P. Golubev, I. K. Kaufman, D. G. Luchinsky, P. V. E. McClintock, and E. A. Zhukov, Appl. Phys. Lett. 67, 308 (1995).

30. P. Jung, Phys. Rep. 234, 175 (1993).

31. N. G. Stocks, N. D. Stein, S. M. Soskin, and P. V. E. McClintock, J. Phys. A 25, L1119 (1992) 
32. F. Moss, in G. H. Weiss, ed., Contemporary Problems in Statistical Physics, SIAM, Philadelphia, 1994, vpp. 205-253.

33. K. Wiesenfeld, and F. Moss, Nature 373, 33 (1995).

34. M. I. Dykman, R. Mannella, P. V. E. McClintock, and N. G. Stocks, Phys. Rev. Lett. 65, 2606 (1990).

35. M. I. Dykman, R. Mannella, P. V. E. McClintock, and N. G. Stocks, JETP Lett. 52, 141 (1990).

36. T. Zhou, F. Moss, and P. Jung, Phys. Rev. A 42, 3161 (1990).

37. M. I. Dykman, R. Mannella, P. V. E. McClintock, N. D. Stein, and N. G. Stocks, Phys. Rev. E 47, 1629 (1993).

38. N. G. Stocks, Nuovo Cimento D 17, 925 (1995).

39. N. G. Stocks, N. D. Stein, and P. V. E. McClintock, J. Phys. A 26, L385 (1993).

40. I. Kh. Kaufman, D. G. Luchinsky, P. V. E. McClintock, S. M. Soskin, and N. D. Stein, Phys. Rev. E 57, 78-87 (1998); I. Kh. Kaufman, D. G. Luchinsky, P. V. E. McClintock, S. M. Soskin, and N. D. Stein, Phys. Lett. A 220, 219-223, (1996).

41. M. A. Ivanov, L. B. Kvashnina, and M. A. Krivoglaz, Sov. Phys. Solid State 7, 1652 (1966).

42. M. A. Dykman, and M. I. Krivoglaz, in M. Khalatnikov, ed., Soviet Physics Reviews, Harwood Academic Publishers, New York, 1984, Vol. 5, pp. 265-442.

43. L. Alfonsi, L. Gammaitoni, S. Santucci, and A. R. Bulsara, Phys. Rev. E 62, 299, (2000)

44. L. Onsager, and S. Machlup, Phys. Rev. 91, 1505 (1953).

45. E. Arimondo, D. Hennequin, and P. Gloriuex, in F. Moss and P. V. E. McClintock, Eds., Noise in Nonlinear Dynamical Systems, Cambridge University Press, Cambridge, 1989, Vol. 3, pp 119-158

46. R. Roy, A. W. Yu, and S. Zhu, in F. Moss and P. V. E. McClintock, Eds., Noise in Nonlinear Dynamical Systems, Cambridge University Press, Cambridge, 1989, Vol. 3, pp 90-118.

47. M. C. Cross, and P. C. Hohenberg, Rev. Mod. Phys. 65, 851 (1993).

48. G. Gabrielse, H. Dehmelt, and W. Kells, Phys. Rev. Lett. 54, 537 (1985).

49. H. Dehmelt, Rev. Mod. Phys. 62, 525 (1990).

50. J. Tan and G. Gabrielse, Phys. Rev. Lett. 67, 3090 (1991); J. Tan and G. Gabrielse, Phys. Rev. A 48, 3105 (1993). 
51. M. O. Magnasco, Phys. Rev. Lett. 71, 1477 (1993).

52. R. D. Astumian, and M. Bier, Phys. Rev. Lett. 72, 1766 (1994).

53. M. M. Millonas, and M. I. Dykman, Phys. Lett. A 185, 65 (1994).

54. J. Prost, J. F. Chauwin, L. Peliti, and A. Ajdari, Phys. Rev. Lett. 72, 2652 (1994).

55. C. R. Doering, W. Horsthemke, and J. Riordan, Phys. Rev. Lett. 72, 2984 (1994).

56. M. I. Dykman, H. Rabitz, V. N. Smelyanskiy, and B. E. Vugmeister, Phys. Rev. Lett. 79, 1178 (1997).

57. A. D. Wentzell and M. I. Freidlin, Russ. Math. Surveys 25, 1 (1970); M. I. Freidlin and A. D. Wentzell, Random Perturbations in Dynamical Systems, Springer, New York, 1984.

58. A. J. McKane, Phys. Rev. A 40, 4050 (1989).

59. R. S. Maier, and D. L. Stein, Phys. Rev. E 48, 931 (1993).

60. M. I. Dykman, P. V. E. McClintock, V. N. Smelyanski, N. D. Stein, and N. G. Stocks, Phys. Rev. Lett. 68, 2718 (1992).

61. M. I. Dykman, E. Mori, J. Ross, and P. M. Hunt, J Chem. Phys. 100, 5735 (1994).

62. D. G. Luchinsky, and P. V. E. McClintock, Nature 389, 463 (1997).

63. R. P. Feynman and A. R. Hibbs, Quantum mechanics and path integrals, McGrawHill, New York, 1965.

64. R. Graham, in G. Höbler, ed., Quantum Statistics in Optics and Solid-State Physics, Springer Tracts in Modern Physics, Springer, Berlin, 1973, Vol. 66, 1-97; R. Graham, and T. Tél, Phys. Rev. Lett. 52, 9 (1984); R. Graham, and T. Tél, Phys. Rev. A 31, 1109 (1985); R. Graham, in F. Moss and P. V. E. McClintock, eds., Noise in Nonlinear Dynamical Systems, Cambridge University Press, Cambridge, 1989, Vol. 1, pp 225-278.

65. M. I. Dykman, and M. A. Krivoglaz, Soviet Phys. JETP 50, 30 (1979).

66. J. F. Luciani, and A. D. Verga, Europhys. Lett. 4, 255 (1987).

67. J. F. Luciani, and A. D. Verga, J. Stat. Phys. 50, 567 (1988).

68. G. Tsironis, and P. Grigolini, Phys. Rev. Lett. 61, 7 (1988).

69. A. J. Bray, and A. J. McKane, Phys. Rev. Lett. 62, 493 (1989).

70. H. S. Wio, P. Colet, M. San Miguel, L. Pesquera, and M. A. Rodríguez, Phys. Rev. A 40, 7312 (1989). 


\section{FLUCTUATIONAL ESCAPE AND RELATED PHENOMENA}

71. A. J. McKane, H. C. Luckock, and A. J. Bray, Phys. Rev. A 41, 644 (1990).

72. A. J. Bray, A. J. McKane, and T. J. Newman, Phys. Rev. A 41, 657 (1990).

73. H. C. Luckock, and A. J. McKane, Phys. Rev A, 42, 1982 (1990).

74. J. K. Cohen, and R. M. Lewis, J. Inst. Math. Appl. 3 266-290 (1967).

75. M. M. Kłosek-Dygas, B. J. Matkowsky, and Z. Schuss, SIAM J. Appl. Math. 48, 425 (1988).

76. M. M. Kłosek-Dygas, B. J. Matkowsky, and Z. Schuss, Phys. Rev. A 38, 2605 (1988).

77. M. M. Kłosek-Dygas, B. J. Matkowsky, and Z. Schuss, J. Stat. Phys. 54, 1309-1320 (1989).

78. M. I. Dykman, Phys. Rev. A 42, 2020 (1990).

79. S. J. B. Einchcomb, and A. J. McKane, Phys. Rev. E 51, 2974 (1995).

80. M. I. Dykman, V. N. Smelyanskiy, D. G. Luchinsky, R. Mannella, P. V. E. McClintock, and N. D. Stein, Int. J. Bifurc. and Chaos 8, 747 (1998).

81. K. Lindenberg, B. J. West, and J. Masoliver, in F. Moss and P. V. E. McClintock, eds., Noise in Nonlinear Dynamical Systems, Cambridge University Press, Cambridge, 1989, Vol. 1, pp 110-160.

82. M. I. Dykman, and K. Lindenberg, in G. H. Weiss, ed., Contemporary Problems in Statistical Physics, SIAM, Philadelphia, 1994, pp 41-101.

83. J. Hales, A. Zhukov, R. Roy, and M. I. Dykman, Phys. Rev. Lett. 85, 78 (2000)

84. M. B. Willemsen, M. P. van Exter, and J. P. Woerdman, Phys. Rev. Lett. 84, 4337 (2000)

85. M. Topaler, and N. Makri, J. Chem. Phys. 101, 7500 (1994).

86. E. W. G. Diau, J. L. Herek, Z. H. Kim, and A. H. Zewail, Science 279, 847 (1998); D. E. Folmer, L. Poth, E. S. Wisniewski, and A. W. Castleman Jr., Chem. Phys. Lett. 287, 1 (1998); A. H. Zewail, Femtochemistry: ultrafast dynamics of the chemical bond, World Scientific, Singapore, 1994.

87. V. N. Smelyanskiy, M. I. Dykman, H. Rabitz, and B. E. Vugmeister, Phys. Rev. Lett. 79, 3113 (1997).

88. L. I. McCann, M. I. Dykman, and B. Golding, Nature 402, 785 (1999).

89. B. Golding, L. I. McCann, and M. I. Dykman, in D. S. Broomhead, E. A. Luchinskaya, P. V. E. McClintock, and T. Mullin, eds., Stochastic and Chaotic Dynamics in the Lakes, American Institute of Physics, Melville, 2000, pp. 34-41. 
90. R. L. Kautz, Rep. Prog. Phys. 59, 935 (1996).

91. M. I. Dykman, R. Mannella, P. V. E. McClintock, S. M. Soskin, and N. G. Stocks, Phys. Rev. A 42, 7041 (1990).

92. R. L. Kautz, Phys. Lett. A 125, 315 (1987).

93. P. Grassberger, J. Phys. A 22, 3283 (1989).

94. R. Graham, A. Hamm, and T. Tél, Phys. Rev. Lett. 66, 3089 (1991).

95. D. G. Luchinsky, J. Phys. A 30, L577 (1997).

96. S. Fauve, and F. Heslot, Phys. Lett. A 97, 5 (1983); B. McNamara, and K. Wiesenfeld Phys. Rev. A 39, 4854 (1989).

97. (a) R. N. Mantegna, and B. Spagnolo, Phys. Rev. E, 49, R1792 (1994); (b) A. D. Hibbs, A. L. Singsaas, E. W. Jacobs, A. R. Bulsara, and J. J. Bekkedahl, J. Appl. Phys. 77, 2582 (1995); A. D. Hibbs, E. W. Jacobs, A. R. Bulsara, J. J. Bekkedahl, and F. Moss, Nuovo Cimento D 17, 811 (1995).

98. L. Gammaitoni, M. Martinelli, L. Pardi, and S. Santucci, Phys. Rev. Lett. 67, 1799 (1991); L. Gammaitoni, F. Marchesoni, M. Martinelli, L. Pardi, and S. Santucci, Phys. Lett. A 158, 449 (1991).

99. E. Ippen, J. Lindner, and W. L. Ditto, J. Stat. Phys. 70, 437 (1993).

100. G. Debnath, T. Zhou, and F. Moss, Phys. Rev. A 39, 4323 (1989); L. Gammaitoni, F. Marchesoni, E. Menichella-Saetta, and S. Santucci, Phys. Rev. Lett. 62, 349 (1989).

101. J. K. Douglass, L. Wilkens, E. Pantazelou, and F. Moss, Nature 365, 337 (1993).

102. Special issue of the J. Stat. Phys. 70, no. 1/2, (1993); special issue of Nuovo Cimento D 17, no. 7-8, (1995).

103. P. J. W. Debye, Polar Molecules, Dover, New York, 1929.

104. G. P. Agrawal, Fiber-optic communication systems, Wiley, New York, 1992.

105. Image and Video Processing II, Proceedings of SPIE, 2182, Bellingham, Washington, 1994; J. W. Bilbo and E. Parks, eds., Quality and Reliability for Optical Systems, Bellingham, Washington, 1993.

106. H. Gibbs, Optical Bistability: Controlling Light with Light, Academic Press, New York, 1985.

107. T. Rivera, F. R. Ladan, A. Israël, R. Azoulay, R. Kuszelevicz, and J. L. Oudar, Appl. Phys. Lett. 64, 869 (1994).

108. M. I. Dykman, G. P. Golubev, D. G. Luchinsky, A. L. Velikovich, and S. V. Tsuprikov, Phys. Rev. A 44, 2439 (1991). 
109. H. Kramers, Physica 7, 284 (1940).

110. L. A. Lugiato, and R. Bonifacio, in F.T. Arecchi, R. Bonifacio, and M.O. Scully, eds., Coherence in Spectroscopy and Modern Physics, Plenum, New York, 1978, p. 85; L. A. Lugiato, Contemp. Phys. 24, 333 (1983).

111. G. P. Golubev, D. G. Luchinsky, A. L. Velikovich, and M. A. Liberman, Opt. Comm. 64, 181 (1987); A. L. Velikovich, G. P. Golubev, V. P. Golubchenko, and D. G. Luchinsky, Opt. Comm. 80, 444 (1991).

112. D. G. Luchinsky, P. V. E. McClintock, and M. I. Dykman, Rep. Prog. Phys. 61, 889 (1998).

113. A. D. Lloyd, I. Janossy, H. A. Mackenzie, B. S. Wherret, Opt. Comm. 61, 339 (1987); D. C. Hutchings, A. D. Lloyd, I. Janossy, B. S. Wherret, ibid, p. 345; P. Pirani, V. Briguet, and W. Lucosz, Helvetica Phys. Acta 60, 836 (1987);

114. M. I. Dykman, G. P. Golubev, I. Kh. Kaufman, D. G. Luchinsky, P. V. E. McClintock, and E. A. Zhukov, Nuovo Cimento D 17, 743 (1995).

115. L. D. Landau, and E. M. Lifshitz, Mechanics, 3rd Edition, Pergamon, Oxford, 1976.

116. Beside [53, 54, 55], see also M. Magnasco, Phys. Rev. Lett. 71, 1477 (1993); R. D. Astumian, and M. Bier, Phys. Rev. Lett. 72, 1766 (1994); F. Marchesoni, Phys. Rev. Lett. 77, 2364 (1996); and references therein.

117. D. Ludwig, SIAM Rev. 17, 605 (1975).

118. H. R. Jauslin, J. Stat. Phys. 42, 573 (1986); Physica 144A, 179 (1987).

119. M. I. Dykman, M. M. Millonas, and V. N. Smelyanskiy, Phys. Lett. A 195, 53 (1994).

120. R. S. Maier, and D. L. Stein, J. Stat. Phys. 83, 291 (1996).

121. R. S. Maier, and D. L. Stein, SIAM J. Apll. Math. 57, 752 (1997).

122. L. S. Schulman, Techniques and Applications of Path Integration, Wiley, New York, 1981.

123. M. I. Dykman, D. G. Luchinsky, P. V. E. McClintock, and V. N. Smelyanskiy, Phys. Rev. Lett. 77, 5229 (1996).

124. S. Wiggins, Global Bifurcations and Chaos: Analytical Methods, SpringerVerlag, New York, 1988.

125. L. Fronzoni, in F. Moss, and P. V. E. McClintock, eds., Noise in Nonlinear Dynamical Systems, Cambridge University Press, Cambridge, 1989, Vol. 3, pp 222-242; P. V. E. McClintock, and F. Moss, in loc. cit., pp 243-274. 
126. M. B. Willemsen, M. P. van Exter, and J. P. Woerdman, Phys. Rev. A 60, 4105 (1999).

127. M. B. Willemsen, M. U. F. Khalid, M. P. van Exter, and J. P. Woerdman, Phys. Rev. Lett. 82, 4815 (1999).

128. M. I. Dykman, V. N. Smelyanskiy, D. G. Luchinsky, R. Mannella, P. V. E. McClintock, and N. D. Stein, Int. J. Bifurc. and Chaos 4, 747 (1998).

129. M. I. Dykman, V. N. Smelyanskiy, D. G. Luchinsky, R. Mannella, P. V. E. McClintock, and N. D. Stein, Nonlinear Phenomena in Complex Systems 2, 1 (1999).

130. D. G. Luchinsky, R. S. Maier, R. Mannella, P. V. E. McClintock, and D. L. Stein, Phys. Rev. Lett. 79, 3109 (1997).

131. D. G. Luchinsky, R. S. Maier, R. Mannella, P. V. E. McClintock, and D. L. Stein, Phys. Rev. Lett. 82, 1806 (1999).

132. B. E. Vugmeister, J. Botina, and H. Rabitz, Phys. Rev. E 55, 5338 (1997).

133. R. Mannella, Phys. Rev. E 59, 2479 (1999); B. E. Vugmeister, J. Botina, and H. Rabitz, Phys. Rev. E 59, 2481 (1999).

134. S. M. Soskin, J. Stat. Phys. 97, 609 (1999).

135. S. M. Soskin, V. I. Sheka, T. L. Linnik, M. Arrayás, I. Kh. Kaufman, D. G. Luchinsky, P. V. E. McClintock, and R. Mannella, in D. S. Broomhead, E. A. Luchinskaya, P. V. E. McClintock and T. Mullin, eds., Stochaos: Stochastic and Chaotic Dynamics in the Lakes, Vol. 502, 60-65, American Institute of Physics, Woodbury, New York, 2000.

136. S. M. Soskin, V. I. Sheka, T. L. Linnik, and R. Mannella, "Short time-scales in the Kramers problem: a step-wise growth of the escape flux", to appear on Phys. Rev. Lett.; M. Arrayás, I. Kh. Kaufman, D. G. Luchinsky, P. V. E. McClintock, and S. M. Soskin, Phys. Rev. Lett. 84, 2556 (2000).

137. P. Hanggi, P. Talkner, and M. Borkovec, Rev. Mod. Phys. 62, 251 (1990).

138. V. I. Mel'nikov, Phys. Rep. 209, 1 (1991).

139. V. A. Shneidman, Phys. Rev. E 56, 5257 (1997).

140. D. G. Luchinsky, R. Mannella, P. V. E. McClintock, M. I. Dykman, and V. N. Smelyanskiy, J. Phys. A 32, L321 (1999).

141. M. Marder, Phys. Rev. Lett. 74, 4547 (1995).

142. R. Mannella, in L. Vázquez, F. Tirando, and I. Martin, eds., Supercomputation in Nonlinear and Disordered Systems, World Scientific, Singapore, 1997, pp. 100-130. 


\section{liv FLUCTUATIONAL ESCAPE AND RELATED PHENOMENA}

143. V. N. Smelyanskiy, M. I. Dykman, and R. S. Maier, Phys. Rev. E 55, 2369 (1997)

144. R. G. Harrison, J. S. Uppal, and P. Osborne, eds., Nonlinear Dynamics and Spatial Complexity in Optical Systems, SUSSP/Institute of Physics, Bristol, 1993.

145. L. A. Lugiato, in E. Wolf, ed., Progress in Optics, North-Holland, Amsterdam, 1984, Vol. 21, pp. 71-211.

146. G. Carpintero, and H. Lamela, Opt. Lett. 24, 1711 (1999).

147. I. B. Schwartz, and T. W. Carr, Phys. Rev. E 59, 6658 (1999).

148. Y. Liu, and P. Davis, Opt. Lett. 25, 475 (2000).

149. T. Shinbrot, E. Ott, C. Grebogi, and A. J. Yorke, Phys. Rev. Lett. 65, 3215 (1990).

150. S. Boccaletti, C. Grebogy, Y. C. Lai, H. Mancini, and D. Maza, Phys. Rep. 329, 103 (2000).

151. T. Shinbrot, Adv. Phys. 44, 73 (1995).

152. T. Shinbrot, C. Grebogi, J. Ott, and E. Yorke, Nature 363, 411 (1993).

153. B. Hubinger, R. Doerner, and W. Martienssen, Phys. Rev. E 50, 932 (1994).

154. E. Barreto, E. J. Kostelich, C. Grebogy, J. Ott, and E. Yorke, Phys. Rev. E 51, 4169-4172 (1995).

155. E. A. Jackson, Chaos 7, 550-559 (1997).

156. S. P. Raj, and S. Rajasekar, Phys. Rev. E 55, 6237-6240 (1997).

157. C. Reyl, L. Flepp, R. Badii, and E. Brun, Phys. Rev. Lett. 67, 267 (1991).

158. R. Roy, T. W. Murphy, T. D. Maier, Z. Gillis, and E. R. Hunt, Phys. Rev. Lett. 68, 1259 (1992).

159. V. S. Anishchenko, A. S. Kopeikin, T. E. Vadivasova, G. I. Strelkova, and J. Kurths, Phys. Rev. E 62 (2000) (in press).

160. A. L. Shilnikov, Computers Math. Applic. 34245 (1997).

161. C. O. Weiss, N. B. Abraham, and U. Hubner, Phys. Rev. Lett 61, 1587 (1988).

162. C. O. Weiss, U. Hubner, N. B. Abraham, and D. Tang Infrared. Phys. Technol. 36, 489 (1995).

163. L. M. Hocking, Optimal Control, Oxford Appl. Math and Computing Science, Clarendon Press, Oxford, 1997. 
164. V. N. Smelyanskiy, and M. I. Dykman, Phys. Rev. E 55, 2516 (1997).

165. Y. R. Shen, The Principles of Nonlinear Optics, Wiley, New York, 1984.

166. S. V. Gonchenko, L. P. Shilnikov, and D. V. Turaev, Computers Math. Applic. 34195 (1997).

167. D. G. Luchinsky, I. A. Khovanov, and P. V. E. McClintock, Prog. Theor. Phys. Suppl. 139, 152 (2000).

168. D. G. Luchinsky, I. A. Khovanov, R. Mannella, and P. V. E. McClintock, Phys. Rev. Lett. 85, 2100 (2000).

169. R. Zwanzig, J. Stat. Phys. 9, 215 (1973).

170. G. Marsaglia and W.-W. Tsang, SIAM J. Sci. Stat. Comput. 5, 349 (1984).

171. D. G. Luchinsky, P. V. E. McClintock, S. M. Soskin, and R. Mannella, Phys. Rev. Lett. 76, 4453 (1996).

172. I. A. Khovanov, D. G. Luchinsky, R. Mannella, and P. V. E. McClintock, "Optimal fluctuations and the control of chaos" submitted to Int. J. Bifurc. Chaos.

173. D. Auerbach, P. Cvitanović, J-P. Eckmann, G. Gunaratne, and I. Procaccia, Phys. Rev. Lett. 58, 2387 (1987).

174. C. Grebogi, E. Ott, and J. A. Yorke, Phys. Rev. A 37, 1711 (1988).

175. P. Schmelcher, and F. K. Diakonos, Phys. Rev. Lett. 78, 4733 (1997).

176. Codes can be obtained from http://www.netlib.no/.

177. J. R. Cash, Comput. Math. Appl. 12A, 1029 (1986); J. R. Cash, SIAM J. Numer. Anal. 25, 862 (1988); J. R. Cash and M. H. Wright, Computing 45, 17 (1990); J. R. Cash and M. H. Wright, SIAM J. Sci. Stat. Comput. 12, 971 (1991).

178. E. N. Lorenz, J. Atmos. Sci. 20, 130 (1963).

179. H. Haken, Phys. Lett. 53A, 77 (1975).

180. C. Sparrow, The Lorenz Equations: Bifurcations, Chaos and Strange Attractors, Springer, New-York, 1982.

181. I. A. Khovanov, D. G. Luchinsky, R. Mannella, and P. V. E. McClintock, in J. A. Freund and T. Pöschel, eds., Stochastic Processes in Physics, Chemistry and Biology, Springer, Berlin, 2000, pp 378-389.

182. M. E. Johnson, M. S. Jolly, and I. G. Kevrekidis, Numerical Algorithms 14, 125 (1997). 